\title{
Hyperactivity of indirect pathway-projecting spiny projection neurons drives compulsive behavior
}

Sean C Piantadosi ${ }^{\star 1,2,3}$, Elizabeth E Manning ${ }^{\star 2,4}$, Brittany L Chamberlain ${ }^{1,2}$, James Hyde ${ }^{2,5}$, Zoe LaPalombara $^{1,2}$, Nicholas M Bannon ${ }^{2}$, Jamie L Pierson ${ }^{2}$, Vijay MK Namboodiri ${ }^{6}$, Susanne E Ahmari ${ }^{1,2 \#}$

Affiliation(s): ${ }^{1}$ Center for Neuroscience, University of Pittsburgh, Pittsburgh PA, USA; ${ }^{2}$ Department of Psychiatry, University of Pittsburgh, Pittsburgh PA, USA; ${ }^{3}$ Department of Anesthesiology and Pain Medicine, University of Washington, Seattle WA, USA; ${ }^{4}$ School of Biomedical Sciences and Pharmacy, University of Newcastle, Callaghan, NSW, Australia; ${ }^{5}$ Department of Biology, Southern Arkansas University, Magnolia AK, USA; ${ }^{6}$ Department of Neurology, University of San Francisco, San Francisco CA, USA.

*These authors contributed equally

\#Corresponding author (Susanne E Ahmari, ahmarise@upmc.edu)

\section{Abstract}

Compulsive behaviors are a hallmark symptom of obsessive compulsive disorder (OCD). Striatal hyperactivity has been linked to compulsive behavior generation in correlative studies in humans and causal studies in rodents. However, the contribution of the two distinct striatal output populations to the generation and treatment of compulsive behavior is unknown. These populations of direct and indirect pathway-projecting spiny projection neurons (SPNs) have classically been thought to promote or suppress actions, respectively, leading to a long-held hypothesis that increased output of direct relative to indirect pathway promotes compulsive behavior. Contrary to this hypothesis, here we find that indirect pathway hyperactivity drives compulsive grooming in the Sapap3-knockout mouse model of OCD-relevant behavior. Furthermore, we show that suppression of indirect pathway activity using optogenetics or treatment with the first-line OCD pharmacotherapy fluoxetine is associated with reduced compulsive behavior. Together, these findings highlight the striatal indirect pathway as a potential new treatment target for compulsive behavior. 


\section{Introduction}

Convergent evidence from structural and functional imaging studies has broadly linked obsessive compulsive disorder (OCD) symptoms to abnormalities in the striatum, specifically highlighting hyperactivity in the caudate at baseline and during symptom provocation ${ }^{1-8}$. Effective treatment with serotonin reuptake inhibitors (SRIs) normalizes striatal hyperactivity, suggesting that this abnormal activity underlies OCD symptoms ${ }^{9,10}$. Complementary findings from rodents provide a causal link between hyperactivity in striatal subregions and generation of compulsive behavior relevant to symptoms of $O C D^{11-14}$. Together, these data strongly implicate modulation of striatal activity in the generation and treatment of compulsive behavior. However, they do not provide insight into how distinct classes of striatal spiny projection neurons (SPNs), which can be defined by their dopamine receptor expression patterns or projection targets in the basal ganglia, contribute to the pathophysiology of OCD. Classically, direct pathway-projecting SPNs (dSPNs) that preferentially express the dopamine D1-receptor are thought to promote movement, while indirect pathway-projecting SPNs (iSPNs) that preferentially express the dopamine D2-receptor are thought to suppress movement ${ }^{15-17}$. Understanding how activity of these specific striatal neuronal populations and their associated pathways through the basal ganglia contribute to the generation of compulsive behaviors may help guide the development of new treatment strategies.

A longstanding theory suggests that OCD symptoms result from increased dSPN activity and/or decreased iSPN activity ${ }^{5,8,18}$. While evidence for this idea has been observed in other models of pathological repetitive behaviors ${ }^{19-24}$, limited support exists in the context of intrinsically generated compulsive behaviors relevant to $O C D^{25,26}$, with no in vivo assessments of activity balance between pathways conducted to date. Here we tested this theory by directly examining activity of SPN subtypes in freely moving Sapap3-knockout (Sapap3-KO) mice, a strain that exhibits SRI-sensitive, OCD-relevant behavioral phenotypes including compulsive grooming behavior, along with well-established structural and physiological striatal abnormalities ${ }^{12,26-33}$.

Using in vivo calcium imaging in Sapap3-KOs, we found that hyperactivity in the central striatum (CS), which has previously been demonstrated to be critical for compulsive grooming in this model ${ }^{12}$, results from an increased number of activated neurons at the onset of compulsive grooming bouts. We then used genetic and 
circuit-based strategies to isolate putative direct and indirect pathway-projecting SPNs. Surprisingly, we found that hyperactivity in the indirect pathway is associated with compulsive grooming, and that a reduction of indirect pathway hyperactivity is associated with decreased compulsive grooming. Together, these results point to an unexpected role for iSPNs in the generation of OCD-relevant compulsive behaviors and suggest new avenues for circuit-based treatment strategies.

\section{Results}

\section{Central striatum is hyperactive during grooming in Sapap3-KO mice}

To investigate the net output of all CS neuron subtypes during compulsive grooming behavior, we expressed the genetically encoded calcium indicator GCaMP6m via human synapsin promoter and implanted a gradient refractive index (GRIN) lens above the injection site (Fig.1a; Fig.S1a). After 5 weeks virus incubation, grooming behavior was assessed and central striatal calcium activity was recorded using a singlephoton miniature microscope during a 40-minute session. Consistent with previous reports ${ }^{12,28,29}$, Sapap3-KO mice spent significantly more time grooming and engaged in significantly more grooming bouts and fewer locomotion bouts than wildtype (WT) littermates (Fig.1c; Fig.S1b). Calcium activity of hundreds of putative central striatal neurons was segmented as previously described (Fig.1d) ${ }^{34,35}$, and a continuous fluorescence time series was extracted for each neuron (see Methods). We first evaluated the degree to which central striatal population activity was able to decode spontaneous grooming displayed by both Sapap3-WT and -KO mice. Using a binary support vector machine (SVM) classifier, we found that central striatal population activity is better able to classify grooming behavior compared to shuffled grooming behavior in both genotypes

(Fig.1e). Furthermore, we observed that grooming behavior was more precisely classified (true positives / (true positives + false positives)) in Sapap3-KOs compared to Sapap3-WTs (Fig.1e), indicating that abnormal central striatal activity may contribute to generation of compulsive grooming.

We next examined when and how central striatal activity was different during compulsive versus normal grooming behavior. CS activity increased transiently at onset of grooming behavior significantly more in Sapap3-KOs compared to -WTs, and this increase persisted throughout grooming bouts (Fig.1f). No difference in locomotion-onset-associated fluorescence was detected between genotypes (Fig.S1c). This grooming- 
onset-associated increase in fluorescence in central striatal neurons was also associated with a significantly elevated calcium event rate in Sapap3-KOs compared to Sapap3-WTs during grooming (Fig.1g; left). In contrast, no difference was observed in the overall calcium event rate during all non-grooming periods (Fig.1g; right), indicating that CS hyperactivity in KOs is restricted to the time of engagement in compulsive grooming behavior. The enhanced predictive strength and CS hyperactivity in Sapap3-KOs could result from a difference in the number of cells active during grooming. Using a statistical method to classify neurons as activated or inhibited at grooming onset (a period -0.5 s before to 3 s after grooming start) ${ }^{36}$, we found that all mice in both genotypes had central striatal neurons with grooming-onset modulated activity (Fig.1h). Compared to WTs, Sapap3-KOs had a significantly greater percentage of grooming-onset activated neurons (Fig.1i,j), while no genotype difference was observed in the percentage of neurons modulated at onset of locomotion (Fig.S1d). The increase in activated neurons at grooming onset in KOs was not due solely to the greater number of grooming bouts, as restricting the number of bouts analyzed in Sapap3-KOs to the mean bout number in WT mice still produced a significant increase in activated neurons (Fig.S1e). No differences were observed in percentage of grooming-onset inhibited neurons (Fig.1j). These data suggest compulsive grooming may result from central striatal hyperactivity driven by increased recruitment of activated SPNs, which in turn may yield stronger population decoding of grooming behavior in KOs.

\section{D1-SPNs are not hyperactive at onset of compulsive behavior}

Given that pharmacological activation of dopamine D1-receptors preferentially expressed by direct pathway-projecting SPNs produces grooming ${ }^{37-40}$ and direct pathway hyperactivity has long been hypothesized to underlie compulsive behavior ${ }^{4,6,8,18}$, we predicted that increased grooming-onset D1-SPN activity would explain the central striatal hyperactivity observed in Sapap3-KOs (Fig.1). To selectively image D1-SPNs, we injected Cre-dependent DIO-GCaMP6m into CS of double transgenic heterozygous D1-Cre ${ }^{+/} /$Sapap3 $^{/-}$and

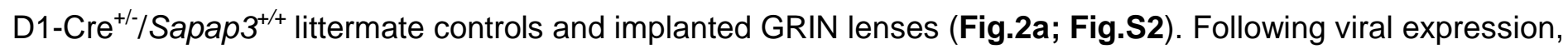
mice underwent a 40-minute grooming and imaging session (Fig.2b). As before, Sapap3-KOs spent significantly more time grooming and engaged in significantly more grooming bouts than Sapap3-WTs (Fig.2c). Surprisingly, we found no significant elevation of D1-SPN calcium fluorescence in Sapap3-KOs 
compared to -WTs at grooming onset (Fig.2d). Similarly, no genotype differences were detected in D1-SPN calcium event rates during either grooming or non-grooming epochs (Fig.2e). Furthermore, WT and KO mice showed no differences in percentage of D1-SPNs that were activated or inhibited at grooming onset (Fig.2f) or the precision of grooming classification by D1-SPN activity (Fig.2g-h). Contrary to our hypothesis, these data suggest that increased CS activity at compulsive grooming onset in KOs is not explained by activity of D1SPNs.

While we did not detect grooming-onset activity differences between Sapap3-KOs and -WTs in the combined D1-SPN population average, we noted that individual neurons displayed substantial heterogeneity in activity profiles (Fig.2d). Because of prior evidence that different activity patterns of individual striatal neurons correspond to specific phases of grooming ${ }^{41,42}$, we next asked if specific subpopulations of D1-SPNs distinguish grooming in Sapap3-KOs vs -WTs. We used an unsupervised clustering algorithm ${ }^{43}$ to group neurons with similar temporal dynamics in their average grooming-onset aligned activity. This approach identified 8 functional subpopulations (a.k.a. clusters) of grooming-related activity (Fig.2i; Fig.S3. Neurons were identified in each of these 8 clusters in each mouse of both genotypes. Clusters were generally separable in high-dimensional principal component space from every other cluster (Fig.S3b-c), supporting the idea that functionally distinct groups of D1-SPNs may differentially control distinct grooming sequence phases in both Sapap3-WT and -KO mice. Several clusters displayed increased activity (above baseline) before (Cluster 2) and at different phases throughout (Clusters 1, 3, 4, 6) grooming behavior in both genotypes. Other clusters displayed reductions in activity during grooming (Cluster 5) or limited grooming-associated changes (Clusters 7 and 8). These data suggest that similar activity of D1-SPN functional clusters occurs during grooming in both WT and KO mice.

While D1-SPN average population activity at grooming onset was not different in Sapap3-WT vs. -KO mice (Fig.2d-f), it is possible that differential recruitment of D1-SPNs within these functional clusters is associated with compulsive grooming. We found that Sapap3-KOs had lower proportions of D1-SPNs that were active prior to grooming onset (Cluster 2) and toward the end of a grooming sequence (Cluster 6; mean grooming bout duration: $\mathrm{KO}=8.2 \mathrm{~s}, \mathrm{WT}=5.3 \mathrm{~s}$ ). Relative to WT mice, Sapap3-KOs also had increased proportions of neurons that were activated shortly after grooming onset (Cluster 3) and that were largely 
unaffected by grooming (Cluster 8). Together these data from functional clusters also did not support our hypothesis that hyperactivity of D1-SPNs at grooming onset is the primary driver of compulsive grooming. Specifically, no change (Cluster 1 ) or even a reduction (Cluster 2) in the proportion of activated neurons was detected in clusters whose activity increased immediately at grooming onset. Instead, differences in proportions of WT and KO D1-SPNs were identified in clusters with activity during, rather than preceding, grooming (Cluster 3 and 6).

\section{D2-SPNs are hyperactive at the onset of compulsive behavior}

Given the surprising finding that D1-SPN activity could not explain striatal hyperactivity associated with compulsive grooming onset (Fig.1), we next investigated whether D2-SPNs might unexpectedly be the primary driver. We generated double transgenic mice by crossing adenosine receptor A2a-Cre (A2a-Cre $\left.{ }^{+/}\right)$mice with Sapap3 $^{+/-}$mice. A2a-Cre ${ }^{+/} /$Sapap3-KO and -WT littermates were injected with a Cre-dependent genetically encoded calcium indicator (DIO-GCaMP6m) to generate selective expression in D2-SPNs, and a GRIN lens was implanted into CS (Fig.3a; Fig.S4). After viral expression, mice underwent a 40-minute grooming observation (Fig.3b). Similar to previous cohorts, Sapap3-KOs spent more time grooming and engaged in significantly more grooming bouts than -WTs (Fig.3c). Strikingly, we found that D2-SPN activity was robustly increased in Sapap3-KOs at onset of grooming relative to -WTs (Fig.3d). D2-SPN calcium event rates during grooming were also significantly increased in Sapap3-KOs while no genotype differences were observed in event rates during non-grooming times (Fig.3e), indicating hyperactivity was restricted to compulsive grooming behavior. Much like our imaging findings in all CS neuron cell types (Fig.1), D2-SPN hyperactivity during compulsive grooming in Sapap3-KOs was associated with increased recruitment of grooming-onset activated D2-SPNs relative to -WTs (Fig.3f). Interestingly, whereas D1-SPN activity was able to predict grooming equally well in Sapap3-KOs and -WTs (Fig.2h), D2-SPN population activity was able to more accurately predict compulsive grooming in KOs compared to normal WT grooming behavior (Fig.3h). Together, these data indicate that D2-SPN hyperactivity is likely the primary source of overall striatal hyperactivity associated with compulsive grooming. 
We next determined whether recruitment of functional clusters of D2-SPNs was different during compulsive KO versus normal WT grooming. D2-SPNs were represented in the same 8 clusters identified within the D1-SPN population (Fig.3i), consistent with prior reports that overall patterns of striatal activity during spontaneous behavior are similar between genetically defined SPN subtypes ${ }^{44,45}$. Compared to WTs, Sapap3-KOs had increased proportions of D2-SPNs with activity increases at the onset of grooming (Cluster 1) as well as activity increases during grooming behavior (Clusters $3 \& 4$ ). In contrast, KOs had reduced proportions of functional D2-SPN clusters that displayed limited grooming-associated changes in activity (Clusters 7 and 8). Relatively few D2-SPNs were classified as exhibiting pre-grooming-onset activity (Cluster 2), and no differences in the proportion of SPNs in this cluster were observed between genotypes (Fig.3j). Overall, these data suggest that hyperactivity in functional clusters of D2-SPNs activated immediately at grooming onset are the primary driver of compulsive grooming behavior.

\section{Optogenetic inhibition of striatopallidal iSPN activity reduces compulsive grooming}

These data highlight that neural activity changes in D2-SPNs are associated with compulsive grooming in Sapap3-KOs and may represent an important treatment target. Because D2-receptors are preferentially expressed on indirect pathway-projecting SPNs, circuit-based neurosurgical treatments targeting the indirect pathway could be an effective treatment strategy for compulsive behavior disorders like OCD. To evaluate this possibility, we used a circuit-specific strategy to selectively manipulate striatopallidal iSPNs projecting to the external segment of the globus pallidus (GPe). To compare this approach to our prior genetic targeting of D2SPNs, we injected a retrograde AAV2-Cre-eGFP into GPe of WT mice and 10 weeks later performed in situ hybridization to examine overlap between retrogradely expressed eGFP and markers for D1- (Drd1a) and D2(Drd2) SPNs in CS (Fig.4a). We found that most eGFP+ neurons (65\%) expressed only Drd2, while a minority (25\%) expressed Drd1a and 10\% expressed both markers (Fig.4b-c). Having established this retrograde strategy as an effective way of interrogating the D2-SPN enriched striatopallidal pathway, we determined whether reducing activity of this circuit affects compulsive behavior. We again injected a retrograde AAV2-CreeGFP bilaterally into GPe prior to injection of Cre-dependent AAV5-CAG-FLEX-ArchT-tdTomato or AAV5Ef1a-DIO-tdTomato control virus into CS of WT and KO mice (Fig.4d). Bilateral optic fibers were then placed 
into CS (Fig.4e; Fig.S5). Following viral expression, mice underwent a grooming session during which they received twenty 30 s periods of constant $532 \mathrm{~nm}$ laser illumination separated by 60 s periods without light (Fig.4f). We found that laser illumination did not affect the number of grooming bouts (Fig.4g-h) or time spent grooming (Fig.S6a) in KOs expressing the tdTomato control virus. In contrast, KOs expressing ArchT displayed a significant reduction in the total number of grooming bouts (Fig.4g-h) and total amount of time spent grooming (Fig.S6a) during laser illumination (30s) compared to the period immediately prior to laser illumination (30s). Inhibition of iSPNs did not alter locomotion in KOs (Fig.S6b-c) and did not affect number of grooming bouts or grooming time in WTs (Fig.S6d). Together, these data suggest that pathway specific inhibition of primarily D2-SPNs (65-75\%), which are hyperactive and differentially recruited in KOs compared to WTs (Fig.3), reduces compulsive grooming behavior.

\section{Chronic fluoxetine treatment is associated with a reduction in striatopallidal SPN hyperactivity}

SRIs are efficacious for treating OCD in humans ${ }^{46-49}$ and reducing compulsive behaviors in mouse models ${ }^{11,50,51}$, including compulsive grooming in Sapap3-KO mice ${ }^{28}$. However, the mechanisms through which SRIs reduce compulsivity are unclear. We therefore tested whether chronic treatment with the SRI fluoxetine could normalize the neural correlates of compulsive behavior in striatopallidal (primarily Drd2-expressing) SPNs-chiefly the increased grooming-associated calcium event rates and increased proportion of groomingonset activated neurons that were observed in D2-SPNs (Fig.3). We again used a circuit-based approach by injecting an untagged retrograde AAV2-Cre unilaterally into GPe and a Cre-dependent AAV9-DIO-GCaMP6m into CS ipsilateral to the GPe injection. A GRIN lens was then placed just above the viral injection in CS (Fig.5a-b; Fig.S7a). Mice underwent a 40-minute baseline imaging session and then received injections of fluoxetine (5 mg/kg, i.p.) for the next 7 days before undergoing a second grooming and imaging session. Unlike prior reports ${ }^{28}$, we did not detect a significant reduction in compulsive grooming behavior in KOs following 7 days of fluoxetine treatment (Fig.S7b-d). We therefore continued treatment using a higher dose $(18 \mathrm{mg} / \mathrm{kg}) \mathrm{via}$ drinking water, which has been shown to achieve blood levels similar to high doses used to treat $O C D^{52}$, and has been previously used by our group to reduce compulsive behavior in Sapap3-KOs ${ }^{14}$. Following 4 weeks treatment, mice underwent another imaging session. Fluoxetine was then removed from all cages for two 
weeks, followed by a washout imaging session (Fig.5c). Consistent with prior reports ${ }^{14}$, chronic fluoxetine treatment significantly reduced the number of grooming bouts in KOs but did not significantly alter time spent grooming (Fig.5d). Fluoxetine treatment did not significantly affect grooming bout duration or locomotion in KOs (Fig.S7e,f). Following washout, the number of grooming bouts in KOs returned to baseline levels (Fig.5d). Fluoxetine did not significantly affect grooming behavior in WTs (Fig.S7h-j).

A trend toward a reduction in calcium event rates during grooming after fluoxetine treatment was observed when cells were averaged within each mouse, with no changes observed in event rates during nongrooming periods (Fig.5e). Given that this retrograde approach was selective for only indirect pathwayprojecting SPNs and therefore lower yield, we also compared all cells across mice grouped by treatment day. We found a significant reduction in grooming-associated calcium event rate after fluoxetine treatment which returned to baseline after washout (Fig.S7g). These data suggest that fluoxetine-mediated reduction of iSPN activity leads to a reduction in compulsive behavior. The link between compulsive behavior and activity of striatopallidal iSPNs was further solidified by observations that fluoxetine treatment reduced the precision of iSPN activity to predict compulsive grooming, and predictive precision increased back to baseline levels following washout (Fig.5g). In addition, we previously identified an increased proportion of activated D2-SPNs during compulsive grooming in KOs compared to WT mice (Fig.3f). Here we find that fluoxetine treatment reduces the percentage of striatopallidal iSPNs activated at the onset of compulsive grooming without affecting percentages of grooming-onset inhibited neurons (Fig.3i-j). A positive correlation was observed between the percent change in grooming bouts and the percent change in activated iSPNs following fluoxetine treatment (Fig.5k), supporting that fluoxetine effects on iSPN activity are involved in therapeutic efficacy. Collectively, these results demonstrate that reducing activation of striatopallidal iSPNs is associated with reduced compulsive behavior and may be a mechanism through which SRls exert their therapeutic effects in OCD.

\section{Discussion}

Although abnormal striatal activity has been consistently associated with compulsive behavior in both human and animal studies, the cellular contributions to these activity patterns is unknown. Here we demonstrate that compulsive grooming behavior in Sapap3-KO mice is associated with increased recruitment of CS neurons 
that are activated at grooming onset. In addition, population activity of CS neurons more precisely classifies compulsive grooming in Sapap3-KOs compared to grooming behavior in -WT littermates (Fig.1). Using a genetic approach to selectively image the two major populations of striatal output neurons, we found that dopamine D1-receptor expressing SPNs did not show compulsive grooming-onset hyperactivity, and population activity of these neurons did not predict grooming in KOs better than WTs (Fig.2). Surprisingly, we instead observed grooming-onset hyperactivity of D2-SPNs in KO mice compared to WTs. This hyperactivity stemmed from an increase in the proportion of D2-SPNs that were activated at the onset of grooming. In addition, D2-SPN population activity better predicted compulsive relative to normal grooming. Using an unsupervised clustering algorithm, we also found that higher proportions of D2-SPNs in KOs were represented in clusters characterized by heightened activity at onset of grooming and throughout grooming (Fig.3).

Given that circuit-based therapies for compulsive behavior, such as transcranial magnetic stimulation (TMS) and deep-brain stimulation (DBS), are promising emerging avenues in severe disease, we next capitalized on a circuit-based approach that would be more amenable to translation into humans ${ }^{53}$. Using a retrograde viral strategy to selectively inhibit indirect pathway-projecting SPNs (which primarily express D2receptors) (Fig.4d-f), we found that optogenetic inhibition of striatopallidal SPNs reduced compulsive grooming in KO mice (Fig.4g-h) without affecting grooming in WTs (Fig.S6d). Using the same circuit-based approach, we found that chronic treatment with a first line OCD therapeutic, fluoxetine, reduced compulsive behavior, striatopallidal SPN hyperactivity, and recruitment of grooming-onset active SPNs (Fig.5). Furthermore, the precision of striatopallidal SPN population activity to predict compulsive grooming behavior was reduced following fluoxetine treatment. Together these results demonstrate that indirect pathway striatopallidal SPN hyperactivity is a primary driver of compulsive behavior in this model system and suggest that strategies aimed at reducing such hyperactivity could be beneficial for treating compulsive behaviors in disorders like OCD.

Striatal hyperactivity is associated with compulsive behavior in humans ${ }^{3-5}$, and treatments that reduce compulsive behavior typically normalize striatal hyperactivity ${ }^{10,54}$. Similar striatal hyperactivity and treatment response profiles have been observed in preclinical studies using a variety of mouse models that display robust compulsive or perseverative behaviors ${ }^{11,12,28,51,55}$. It has long been theorized that hyperactivity of the basal ganglia direct pathway (or reduced activation of the indirect pathway) might drive compulsive behaviors, 
including those observed in $O C D^{5,6,8,18}$. Studies in rodents have provided some support for this type of pathway imbalance in the generation of qualitatively similar aberrant behavioral phenotypes, including dyskinesia ${ }^{20-24}$ and compulsive drug seeking ${ }^{56-58}$. Our results suggest that this heuristic is not accurate for compulsive grooming behavior, with hyperactivity instead resulting from increased proportions of grooming-onset activated D2-SPNs and not D1-SPNs in KO mice. One notable difference between compulsive grooming and behaviors such as dyskinesia and compulsive drug seeking (in which a D1-SPN-driven imbalance toward the direct pathway is observed) is that grooming is typically a highly stereotyped, serially ordered behavior ${ }^{41,42,59}$. Critically, compulsive grooming behavior in $\mathrm{KO}$ mice contains the same sequences and motor components as grooming in WTs, but the frequency, duration, and organization of these sequences is disturbed ${ }^{60}$. This disturbance has profound ramifications for a mouse's well-being, resulting in facial lesions and disrupted sleep $^{28}$ as well as impaired ability to mate and care for pups (unpublished observations, Ahmari laboratory). Thus, similar to compulsive behavior in humans with OCD (e.g. compulsive hand-washing in an individual with a contamination obsession), compulsive grooming behavior in $\mathrm{KO}$ mice represents an excessive, repetitive version of a normal behavior that interferes with normal activities ${ }^{13}$.

We used an unsupervised clustering analysis to further examine the patterns of neural activity associated with the onset and maintenance of grooming behavior in both genotypes. Heterogenous response profiles were observed across the population in both D1-SPNs and D2-SPNs mirroring previous work ${ }^{44,45}$, highlighting the importance of coordinated activity between these populations in the normal control of behavior. Across the 8 clusters identified for D2-SPNs, KO mice showed increased proportions of clusters comprised of neurons with increased activity at the onset of grooming (Clusters 1 and 3 ) and late in grooming bouts (Cluster 4), suggesting that striatal hyperactivity during grooming is driven by these grooming-activated clusters. In contrast, D1-SPNs showed mixed changes in cluster identity across genotypes, with KOs showing decreased proportions of grooming onset (Cluster 2) and offset (Cluster 6) activated neurons, but increased proportions of some other grooming-onset activated neurons (Cluster 3) as well as neurons that were inactive during grooming (Cluster 8). Taken together with our observations of no change in grooming-onset fluorescence and grooming-associated calcium event rates in D1-SPNs (Fig.2d-e), these clustering observations suggest that D1-SPNs do not drive striatal hyperactivity associated with grooming onset in KOs. However, increases in the 
proportion of both D1- and D2-SPNs that are active following the onset of grooming bouts (Cluster 3 ) in KO mice may suggest that coordinated activity of these populations supports some aspect of compulsive grooming; this question can be elucidated in future studies. Studies examining striatal control of sequential behavior appear to shed light on our finding that D2-SPN hyperactivity promotes compulsive grooming in Sapap3-KOs. Optogenetic activation of D2-SPNs (as well as inhibition of D1-SPNs) reduced the ability of wildtype mice to complete sequences on a well-trained serial order task ${ }^{61}$, instead leading to an increase in perseverative sequence start responses. This raises the possibility that suppression of D2-SPN activity is necessary for successful completion of action sequences in WT mice. Conversely, D2-SPN hyperactivity might promote compulsive behavior typified by excessive grooming sequence initiation combined with the inability to complete serial grooming bouts known as syntactic grooming chains. This interpretation is supported by ours and others' data that KO mice engage in many more individual grooming bouts than WT mice ${ }^{14,28,62}$. We expand on these observations by identifying a neural substrate through which reducing D2-SPN hyperactivity might exert its anti-compulsive effects-i.e., decreasing recruitment of functional clusters of grooming-onset activated iSPNs (Fig.3). Furthermore, we demonstrate that an effective treatment for OCD suppresses activity of iSPNs that preferentially express D2-receptors, providing the first evidence that this may be an important therapeutic mechanism for the treatment of OCD.

While recently published work supports the idea that inhibiting genetically defined D2-SPNs reduces compulsive grooming behavior in Sapap3-KOs ${ }^{55}$, this type of manipulation is unlikely to be amenable to translation, since treatment with drugs targeting the D2-receptor produces substantial unwanted side effects ${ }^{63}$. In contrast, our circuit-based approach to inhibiting D2-SPNs may more readily inform translation into humans in the form of TMS or DBS. Using a retrograde strategy to infect GPe-projecting SPNs, we found that striatopallidal projections from the associative (central) striatum are comprised of $75 \%$ Drd2-expressing SPNs and 35\% Drd1a-expressing SPNs (10\% express both Drd2 and Drd1a). To our knowledge this is the first quantitative assessment of striatopallidal SPN identity conducted in the associative striatum, though evidence for pallidal innervation by D1-SPNs has previously been found ${ }^{64,65}$. Our findings are consistent with previous functional work demonstrating innervation of the GPe by a small percentage of D1-SPNs $(2 / 11 \text { neurons })^{66}$. Together with previous findings, our work also supports a dorsal/ventral gradient in pallidal innervation by 
striatal D1- and D2-SPNs, with roughly $50 \%$ of ventral striatal D1-SPNs innervating ventral pallidum ${ }^{66}$, while only $\sim 25 \%$ of central striatal D1-SPNs innervate the GPe (Fig.4a-c). Our findings are also in agreement with previous work in the ventral striatum indicating that innervation of the pallidum by D1-SPNs cannot simply be accounted for by SPNs that express both Drd2 and Drd1 ${ }^{66}$. Importantly, our demonstration that a high proportion (25\%) of striatopallidal SPNs in the central striatum do not express D2-receptors emphasizes the need to explore pathway specific manipulations rather than solely genetic strategies to better understand how compulsive behavior is generated.

A key question raised by our experiments is what drives striatopallidal, D2-SPN hyperactivity in Sapap3-KO mice? In wildtype mice, activation of cortical inputs to the striatum preferentially increases immediate-early gene expression in indirect pathway-projecting striatal neurons ${ }^{67,68}$. Numerous studies demonstrate that the associative and sensorimotor striatum of $\mathrm{KO}$ mice receive altered drive from several cortical regions ${ }^{27,28,31}$, including the lateral orbitofrontal cortex (IOFC) and supplementary motor region (M2; also known as anterolateral motor cortex; ALM) ${ }^{12,32,69}$. We have shown that drive from M2 to both central striatal SPNs and fast-spiking interneurons (FSIs), which strongly inhibit nearby SPNs ${ }^{70-72}$, is significantly increased in $\mathrm{KO}$ mice relative to $\mathrm{WTs}^{32}$. Activating this projection promotes naturalistic grooming sequences in WT mice and elicits activation of central striatal SPNs on the same time scale ${ }^{73}$, though the precise striatal cell types affected in vivo are not known. Given that striatal FSIs in the dorsal striatum more strongly inhibit D1SPNs over D2-SPNs ${ }^{74}$, it is plausible that increased drive to FSIs in KO mice may lead to imbalanced output whereby greater proportions of striatonigral SPNs (majority D1-expressing) are strongly inhibited compared to striatopallidal SPNs (majority D2-expressing). Here we demonstrate that enhancing inhibition of striatopallidal SPNs via direct optogenetic inhibition or reducing the proportion of grooming-onset activated striatopallidal SPNs via chronic fluoxetine treatment reduces compulsive behavior, perhaps by compensating for decreased FSI-mediated D2-SPN inhibition in KO mice. Fluoxetine also increases excitability of cortical FSIs ${ }^{75}$, and serotonin increases excitability of striatal $\mathrm{FSIs}^{76}$, suggesting possible indirect mechanisms by which SRIs can impact recruitment of large groups of SPNs. More direct pharmacological manipulations of D2-receptors, including treatment with the partial agonist aripiprazole, have shown some efficacy for augmenting SRI therapy in some treatment refractory OCD subjects ${ }^{77}$. Interestingly, aripiprazole was recently shown to reduce primarily 
short-duration grooming bouts (but not syntactic grooming sequences) and tic-like behaviors in Sapap3-KO mice $^{60}$. Future work is needed to determine the precise circuit and molecular mechanisms by which SRls reduce the recruitment of striatopallidal SPNs.

In conclusion, our work experimentally demonstrates the surprising finding that, contrary to long-held hypotheses, compulsive behavior is driven by hyperactivity of Drd2-expressing, striatopallidal SPNs. Furthermore, we show that the first-line pharmacotherapy for treatment of OCD, fluoxetine, reduces striatopallidal SPN hyperactivity, providing a potential novel mechanism for the treatment of compulsive behavior. 


\section{Methods}

Animals: All procedures were carried out in accordance with the guidelines for the care and use of laboratory animals from the $\mathrm{NIH}$ and with approval from the University of Pittsburgh Institutional Animal Care and Use Committee (IACUC). Sapap3-knockout (Sapap3-KO) and wildtype (WT) littermates expressing the Crerecombinase (Cre) transgene were generated by breeding Sapap3 heterozygous mutants $\left(\right.$ Sapap $\left.{ }^{+-}\right)$with heterozygous D1-Cre mice $\left(\mathrm{D} 1-\mathrm{Cre}^{+-}\right)$or heterozygous A2a-Cre mice $\left(\mathrm{A} 2 \mathrm{a}-\mathrm{Cr} \mathrm{e}^{+/-}\right)^{78}$. Subsequent Sapap3 ${ }^{+/-}$ $:: \mathrm{Cre}^{+/-}$(either D1-Cre ${ }^{+-}$or A2a-Cre ${ }^{+/-}$) mice were mated with a Sapap3 ${ }^{+/-}$mouse, allowing for the generation of

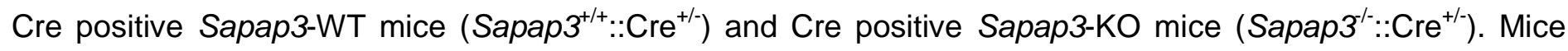
were maintained on a 100\% C57BL/6 background. Male and female Sapap3-KO and wildtype littermates were used for all experiments. Mice in all cohorts were approximately 4-8 months old at the time of initial surgery.

General stereotactic surgical methods: For all surgeries, mice were anesthetized using $5 \%$ isoflurane mixed with oxygen and maintained on $1-2 \%$ isoflurane for the duration of surgery on a small-animal stereotactic instrument (Kopf Instruments). All measurements were made relative to an interpolated bregma. Viral injections were performed using a fixed needle Hamilton syringe (Cole-Parmer Scientific, Vernon Hills IL, USA) connected to sterile polyethylene tubing affixed to a metal cannula and a Harvard Apparatus 11 Elite Syringe Pump (Harvard Apparatus, Holliston MA, USA). Stability of the implant was ensured by placement of one or two $0.45 \mathrm{~mm}$ skull screws secured just in front of the lambdoid suture. Following completion of each surgery, mice were injected with subcutaneous (s.c.) carprofen $(10 \% \mathrm{w} / \mathrm{v}$ in $0.9 \%$ saline) and administered topical antibiotic ointment (TAO) and lidocaine around the headcap. For all surgical procedures mice were kept group housed with littermates unless conspecific fighting was noted, in which case the aggressor was isolated for the duration of the experiment.

In vivo microendoscopy surgical methods: For experiments recording from all central striatal (CS) neuron

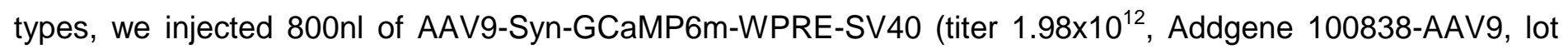
v28555) into CS (AP: +0.65, ML: -1.8, DV: -2.9 \& -3.0). For experiments in CS in D1-Cre / Sapap3 mice and A2a-Cre / Sapap3 mice, 800nl of a virus encoding Cre-dependent GCaMP6m (AAV9-Syn-Flex-GCaMP6mWPRE-SV40, titer $2.72 \times 10^{12}$ ) was injected into CS (AP: +0.65, ML: -1.8, DV: -2.9 \& -3.0). To selectively image striatopallidal neurons in CS, 400nl of a Cre-expressing virus that is retrogradely transported back one synapse 
(AAV-pmSyn1-EBFP-Cre, titer $1.00 \times 10^{13}$, Addgene 51507-AAVrg, lot 27021) was injected into external segment of globus pallidus (GPe; AP: +0.15 , ML: -2.00 , DV: -3.80 ) and 800nl of Cre-dependent GCaMP6m (AAV9-Syn-Flex-GCaMP6m-WPRE-SV40, titer $2.7 \times 10^{12}$ ) was injected into CS (AP: +0.75, ML: -1.80, DV: -2.9 \& -3.2). CS injections were done in a two-step manner, with 400nl of virus injected at DV -2.9 and $400 \mathrm{nl}$ injected at DV -3.0. For all imaging experiments, a 500 $\mu \mathrm{m}$ diameter, $6 \mathrm{~mm}$ length gradient refractive index lens (ProView GRIN lens, Inscopix Palo Alto, CA USA) was lowered just dorsal to viral injection target (AP: +0.65, ML: -1.8, DV: -2.85) (DV: -2.80 in retroAAV GPe experiment) for visualization of cells in the target region. For all GRIN lens implants, acrylic dental cement (Lang Dental, Wheeling IL, USA) was used to secure the lens in place and seal the entire lens during virus incubation period (4-6 weeks).

After 4-6 weeks for viral incubation, mice were again anesthetized with isoflurane and secured to a stereotactic apparatus using ear cuffs. Using a Dremel, excess dental cement was carefully removed to expose ProView GRIN lens. A magnetic microscope baseplate (Part ID:1050-002192, Inscopix) was then attached to the miniaturized microscope (nVistaHD 2.0 epifluorescence microscope, Inscopix) and lowered into place above GRIN lens with 475nm blue LED gain and power set to maximum. Optimal field of view was determined by focusing on visible cells or other gross landmarks (blood vessels). Baseplate was then cemented in place and plastic Microscope Baseplate Cover (Part ID:1050-002193; Inscopix) was attached.

Optogenetic inhibition of striatopallidal SPNs surgical methods: A dual-virus retrograde transfection technique was used to target opsin expression selectively in CS striatopallidal SPNs. 350nl of retrograde Cre (AAVrg-hSyn-Cre-EGFP, titer $1.3 \times 10^{13}$, Addgene 105540-AAVrg, lot v38984) was injected bilaterally into GPe (AP: $+0.15, \mathrm{ML}:-2.00 /+2.00$, DV: -3.80$)$ and 400nl of Cre-dependent archaerhodopsin (400nl pAAV5-FLEXArchT-tdTomato, titer $1.30 \times 10^{13}$, Addgene 28305, lot v24870) or tdTomato (AAV5-Ef1a-DIO-tdTomato, titer $7.3 \times 10^{12}$, UNC viral vector core) were injected bilaterally into CS (AP: +0.75, ML: $\left.-1.80 /+1.80, \mathrm{DV}:-2.9 \&-3.2\right)$ of experimental and control groups, respectively. Optic fibers (200um diameter, 0.39 NA) were implanted bilaterally in CS (AP: +0.75 , ML: $-1.80 /+1.80$, DV: -3.00$)$.

Drug preparation and administration: ( \pm )Fluoxetine hydrochloride (fluoxetine) was obtained through $\mathrm{NIMH}$ Chemical Synthesis and Drug Supply Program. Fluoxetine was first administered (5mg/kg in saline i.p.) once daily for 7 consecutive days as previously described; however, this did not significantly impact compulsive 
grooming behavior (Fig.S7a-C). In the same animals, fluoxetine was next administered via drinking water at higher dose according to established methods ${ }^{11}$. Briefly, bottles were placed in each cage and drinking was monitored for 3 consecutive days. Based on average daily consumption and average weight of mice in each cage, $18 \mathrm{mg} / \mathrm{kg}$ of fluoxetine hydrochloride was mixed with autoclaved drinking water and stored in black bottles to prevent degradation. Drinking was monitored daily, and amounts were adjusted as needed to maintain average dose of $18 \mathrm{mg} / \mathrm{kg}$ fluoxetine per cage. Each cage was given 100mg/L of drinking water. Bottles were changed every 4 days to prevent degradation of the fluoxetine. Body weight was monitored 3x per week. After 4 weeks of administration, fluoxetine was removed from cages and mice were given a two-week washout to monitor whether grooming behavior and neural activity differences returned to baseline levels.

Behavioral apparatus and assessment of grooming behavior and locomotion: A custom-built behavioral apparatus was constructed for delivery of optogenetic stimuli and accurate simultaneous assessment of spontaneous (e.g. grooming) behavior and neural activity via in vivo calcium imaging. A clear plexiglass sheet was suspended over a behavioral acquisition camera (Point Grey Blackfly, FLIR Integrated Imaging Solutions). A clear acrylic chamber (8"x8"x12") was placed above the camera. For calcium imaging, behavioral acquisition was conducted at $40 \mathrm{~Hz}$ using SpinView (Point Grey) software and detailed frame information was sent directly to a central data acquisition box (LabJack U3-LV, Labjack Corporation, Lakewood CO USA). A randomly flashing (30s ITI) LED visible in the behavioral video (controlled by custom scripts via an Arduino (Arduino Leonardo, Somerville MA, USA)) and sending TTL pulses to the LabJack was used for alignment of behavior and calcium data. For optogenetics, behavioral acquisition was conducted at $40 \mathrm{~Hz}$ using SpinView software, and an Arduino controlling laser stimulation also controlled a visible LED in the field of view to synchronize optogenetic stimulation periods to behavior.

Following acquisition, video was converted and compressed (maintaining accurate frame rate information) into .MP4 format using the open-source software HandBrake. Videos were then imported into Noldus The Observer XT (Noldus, Leesburg VA, USA) and grooming behavior was scored frame by frame. Grooming behavior was scored according to previous reports ${ }^{14,59}$ by an observer blind to experimental condition (genotype and drug treatment). Despite advances in machine learning techniques for automated behavioral analysis of grooming, accuracy remains lower than manual grooming scoring. A mouse was 
considered to be grooming if it engaged in any of the following behaviors: 1) Facial grooming: touches its face, whiskers, or head with its forepaws; 2) Body grooming: licks its flank or its ventral surface; 3) Hind leg scratching: uses one of its hind legs to scratch its flank, neck or head. The beginning of a grooming bout was defined as the frame when a mouse made a movement to begin grooming (e.g. a face grooming bout began the frame a mouse lifted its paw off the ground to touch its face). The end of a grooming bout was defined as the frame when a mouse ceased grooming (e.g. a body grooming bout ended when mouse moved its snout from its flank). Consecutive grooming bouts separated by less than one second were collapsed into the previous bout. Thus, the minimum amount of time possible between grooming bouts for all experiments was one second.

For locomotion assessment, behavior videos were imported into Noldus EthoVision XT (Noldus, Leesburg VA, USA). $40 \mathrm{~Hz}$ video files were used for analysis. A square arena was drawn encompassing the entire behavioral chamber filmed from below. Gray-scaling was used to automatically detect a mouse's location within the arena. Within a single experiment, gray-scaling values remained constant across mice. Distance (centimeters), velocity $(\mathrm{cm} / \mathrm{s})$, and $X / Y$ location in space was tracked. For imaging experiments, movement/locomotion onset was identified when a mouse's acceleration exceeded $2 \mathrm{~cm} / \mathrm{s}$. A 5 frame averaging window was used to minimize outliers.

In vivo calcium imaging in freely moving mice: After at least 1 week recovery from baseplate surgery, mice were habituated to attachment of the microscope. Mice were lightly scruffed and the miniature nVistaHD 2.0 microscope was connected to the magnetic baseplate and secured with a set screw. During habituation, optimal focus, field of view, and LED power and gain settings were determined visually by assessing presence of clearly defined putative neurons. A caliper was used to measure the microscope focus to ensure multiple imaging sessions were conducted with same field of view. Mice were also habituated via placement into the acrylic chamber under low light conditions for 5-10 minutes daily for three days prior to imaging.

Following habituation, mice were given a 40-minute baseline behavior and imaging session. Under low light, the microscope was attached, and mice were placed into a temporary holding cage. Mice were given 3-5 minutes after scope attachment for recovery from scruffing and to allow any rapid photobleaching to occur. Mice were then carefully placed into clear acrylic chamber. LabJack data acquisition then began, immediately 
followed by behavioral SpinView recordings and nVistaHD software recording compressed greyscale tiff images at $20 \mathrm{~Hz}$. As with behavioral frame acquisition, individual calcium frame information was sent to LabJack for subsequent alignment of behavior and calcium data. Analog gain of image sensor was set between 1 and 4 and 470 nm LED power was set between 10-60\% transmission range. For retrograde-Cre CS imaging, settings were kept consistent for each mouse throughout all fluoxetine and washout imaging sessions.

Optogenetic inhibition of striatopallidal SPNs during spontaneous behavior: Mice were habituated to attachment of optical patch cables in the behavioral chamber under low light conditions for 5 consecutive days prior to experiment $(5 \mathrm{~min})$. The interface between patch cable and fiberoptic ferrule was covered in black heatshrink during the experiment to minimize light leak. Mice were connected to patch cables and placed in experimental chambers. Mice were given 3-5 minutes between connecting patch-cords and beginning the optical inhibition protocol. Mice received twenty 30 s periods of continuous $30 \mathrm{~mW}$ stimulation from a green 532nm fiber-coupled high-power laser (Opto Engine, Midvale Utah USA) separated by 60s periods without light stimulation.

Histological confirmation of virus, fiber, and GRIN lens/fiber optic probe targeting: Mice were transcardially perfused with $4 \%$ paraformaldehyde (PFA) and phosphate buffered saline (1X PBS). Immediately after perfusion, heads (with implants intact) were placed into $4 \%$ PFA for $24 \mathrm{~h}$ for post-fixation after which brains were removed and transferred to a $30 \%$ sucrose (in 1 X PBS) solution. Brains were frozen and 35um sections were cut on a cryostat in 1:6 series. 1 series was mounted, counterstained with DAPI mounting media (Fluoroshield, Sigma-Aldrich) and imaged at 10x resolution on slide scanning microscope (Olympus VS120) to visualize virus and implant. For experiments imaging striatopallidal neurons using AAVretropmSyn1-EBFP-Cre, a standard immunohistochemical protocol was used to enhance weak native fluorescence [mouse a-Cre recombinase primary antibody (1:1500, MAB3120, Millipore); biotinylated anti-mouse secondary antibody (1:250 Jackson lab); tertiary streptavidin conjugated Cy-3 (1:250 Jackson lab)].

In situ hybridization of striatal SPNs projecting to GPe: RNAscope was performed on fresh frozen striatal slices taken from Sapap3-WTs $(n=5)$ injected bilaterally in Gpe with a retrogradely transported Cre-expressing AAV (350nl AAVrg-hsyn-Cre-eGFP, titer 1.3 x 10^13, Addgene 105540) (AP: -0.3, ML: \pm 1.98, DV: -3.2). 
Tissue was collected 10 weeks after viral injection to allow time for retrograde transport and viral expression. $18 \mu \mathrm{m}$ coronal sections were cut and mounted to slides. In situ hybridization was performed according to RNAscope Multiplex Fluorescent Reagent Kit v2 protocol (Catalog Number 320393) using the following probes: D2 (RNAscope® Probe-Mm-Drd2-C3, catalog \#406501), D1 (RNAscope® Probe-Mm-Drd1a, catalog \#406491), and eGFP (RNAscope® Probe-Mm-EGFP-C2, catalog \#400289). Images were acquired using Olympus Fluoview 3000 confocal microscope (20x magnification). Two blinded experimenters were provided with images of CS sections (300um x 300um region) in which Drd1a and Drd2 were randomly pseudocolored for different experimental subjects, such that experimenters did not know which color corresponded to which probe. Cells projecting from CS to Gpe were labeled with the EGFP probe. Experimenters individually counted the number of individual EGFP+ cells co-labeled with Drd1a, Drd2, or both, for each sample.

Calcium imaging data processing: All imaging pre-processing was performed using Mosaic software (version 1.2.0, Inscopix) via custom Matlab (MATHWORKS, Natick MA, USA) scripts. Videos were spatially downsampled by a binning factor of 4 (16x spatial downsample) and temporally downsampled by a binning factor of 2 (down to 10 frames per second). Lateral brain motion was corrected using the registration engine TurboReg $^{79}$, which uses a single reference frame to match the $X Y$ positions of each frame throughout the video. Motion corrected $10 \mathrm{~Hz}$ video of raw calcium activity was then saved as a .TIFF and used for cell segmentation.

Using custom Matlab scripts, the motion corrected.TIFF video was then processed using the Constrained Non-negative Matrix Factorization approach (CNMFe), which has been optimized to isolate signals from individual putative neurons from microendoscopic imaging ${ }^{34}$. Putative neurons were identified and manually sorted by an observer blind to genotype according to previously established criteria and as per our published work $^{14,35}$. For all analyses on fluorescence traces, the non-denoised temporal traces (referred to as the "raw" trace in CNMFe) without any deconvolution were used. For each individual cell, the raw fluorescence trace was Z-scored to the average fluorescence and standard deviation of that same trace. Thus, fluorescence units presented here are referred to as "Z-scored fluorescence" unless otherwise noted. For analyses of calcium events, calcium events were directly extracted from CNMFe (neuron.S) and binarized. 
Calcium imaging and behavior alignment: Custom Matlab (MATHWORKS) scripts were used to conduct analysis of grooming-related calcium activity. Grooming behavior (state events) was exported as timestamps (grooming start and grooming stop), defined as described above, and aligned to calcium time by recording 5 consecutive pulses of the randomly flashing LED (point events). The offset of Noldus behavior time to nVista calcium time was then subtracted off, leaving the same number of frames for both the behavior and calcium fluorescence. Grooming timestamps were then transferred to a binary/continuous trace of the same length and sampling rate $(10 \mathrm{~Hz})$ as each calcium trace via logical indexing (grooming $=1$, not-grooming = 0 ). Timestamps for behavior are converted to the closest matching frame in the calcium recording (maximum error of one frame or $\pm 100 \mathrm{~ms}$ at $10 \mathrm{~Hz}$ ). Calcium activity could then be aligned to the start and end of a grooming bout.

Analysis of calcium events and calcium fluorescence: For analyses comparing the effect of genotype on binarized calcium event rates, the average calcium event rate per cell was obtained for all grooming and nongrooming periods. The average calcium event rate was then calculated across all cells in a given mouse during each of these periods to obtain a single calcium event rate value for each animal unless otherwise stated. Calcium event rates during grooming and non-grooming periods in WT and KO mice were then compared with independent samples $t$-tests unless otherwise stated. In experiments involving fluoxetine administration, a repeated measures one-way ANOVA was used to analyze calcium event rate changes within each genotype. Sidak's post-hoc correction was used for multiple comparisons. For analysis of grooming-onset aligned calcium fluorescence, each individual cell's activity across all grooming bouts (-3s before grooming onset, +10 s after grooming onset, 130 total samples at $10 \mathrm{~Hz}$ ) was averaged within an individual session. Grooming- and locomotion-onset aligned calcium fluorescence was then averaged across all cells within a single genotype. A paired $t$-test was then conducted comparing the fluorescence at each sample between genotypes. To account for multiple comparisons, a conservative Bonferroni correction was used such that the $\alpha$-value was $=0.00038$ (0.05 / 130). Thus, a significant difference in calcium fluorescence between WT and KO mice for a given sample must obtain a $p$-value of $\leq 0.00038$ (MATLAB).

Event related activity classification: In order to perform unbiased classification of an individual cell's responsiveness (activated, inhibited, or unaffected) to a behavioral event (e.g. grooming or locomotion onset) 
we adapted a previously used strategy ${ }^{36}$. For each individual cell, raw calcium traces 10 seconds prior to grooming onset and 10 seconds after grooming onset (200 total samples at $10 \mathrm{~Hz}, 100 \mathrm{~ms}$ per sample) were shuffled in time for each sample (200x) removing any temporal information that was previously in each trace but maintaining the variance within each grooming bout. This shuffle was then performed 1000 times per cell to obtain a null distribution of grooming associated calcium activity. A cell was considered responsive to grooming onset if its average behavioral event Z-normalized calcium fluorescence amplitude between -0.5s before grooming onset to 3s after grooming onset exceeded a 1 standard deviation threshold from the null distribution. Independent samples $t$-tests were used to compare the proportions of modulated cells between genotypes and drug treatment using GraphPad Prism 8.0 and 9.0 (GraphPad Software, San Diego CA, USA).

Grooming decoding based on CS population activity: Using custom MATLAB scripts, a cubic Support Vector Machine (SVM) classifier was trained using the Z-scored fluorescence signal for the entire session from each neuron within a single mouse. Fluorescence signal (predictors) from each neuron was partitioned (cvpartition) such that the classifier was trained on $75 \%$ of the data and tested on the remaining $25 \%$. A cubic SVM (fitcsvm using PolynomialOrder = 3) was then trained on these data for each mouse. The response variable was a mouse's binary grooming vector. Separate models were trained in which the calcium fluorescence for each neuron was randomly shuffled (randperm) with no repeats. Given that grooming is a relatively infrequent behavior, comparisons of classifier performance across groups used precision [true positive $(T P) / T P+$ false positive $(F P)]$ rather than accuracy $[T P+$ (true negative) $T N / T P+F P+$ false negative $(\mathrm{FN})+\mathrm{TN}]$, which is more easily affected by class imbalance. Classifier precision for both grooming and the shuffled control was compared across genotypes using a one-way or repeated measures ANOVA followed by post-hoc comparisons using Sidak's multiple comparison correction using GraphPad Prism.

Spectral cluster analysis of grooming-related activity: For spectral clustering, each individual cell's activity across all grooming bouts (-3s before grooming onset, +10 s after grooming onset, 130 total samples at 10hz) was averaged within an individual session. Grooming-onset aligned calcium fluorescence was then averaged across all cells within a single genotype. These grooming-onset aligned calcium traces were then concatenated across both genotype (WT and KO) as well as cell type (all SPNs, D1-SPNs, and D2-SPNs). Spectral clustering on this combined vector was performed according to previously established methods using 
custom Python code ${ }^{43}$. Briefly, dimensionality was reduced using principal component analysis (PCA). The number of principal components kept was determined using the standard bend/elbow method in the plot of explained variance (Fig.S3a). Based on this metric, 7 principal components were kept for analysis. Data were then projected into the lower dimensional subspace formed by the principal components and then input into the clustering algorithm ${ }^{43}$. Clustering was performed using the Scikit-learn function sklearn.cluster.Spectralclustering with the affinity matrix calculated using a $k$-nearest neighbor connectivity matrix. The optimal number of clusters was determined by maximizing the silhouette score over a grid search over parameters. Stability of clustering was estimated by subsampling various fractions of trials and calculating the adjusted Rand index. These analyses demonstrated that 8 distinct functional clusters existed in our data. Following clustering, the cluster identity of each individual cell was then mapped back on to its genotype (WT or KO), cell-type (all SPNs, D1-SPNs, and D2-SPNs), and mouse for subsequent analysis. The proportion of neurons in each cluster was compared between genotypes using Chi-squared test (MATLAB).

Statistical analysis of grooming behavior: Grooming behavior was analyzed using two-tailed independent samples $t$-tests to compare genotypes and optogenetic manipulations. For evaluating the effect of fluoxetine treatment on grooming behavior, one-way repeated measures analysis of variance (ANOVA) was used. Main effects and interactions are reported and in the case of significant interactions, post-hoc comparisons were made using Sidak's multiple comparison correction. A corrected alpha was set to 0.05 . Analyses were conducted using GraphPad Prism. 
bioRxiv preprint doi: https://doi.org/10.1101/2022.02.17.480966; this version posted February 18, 2022. The copyright holder for this preprint (which was not certified by peer review) is the author/funder, who has granted bioRxiv a license to display the preprint in perpetuity. It is made available under aCC-BY-NC 4.0 International license.
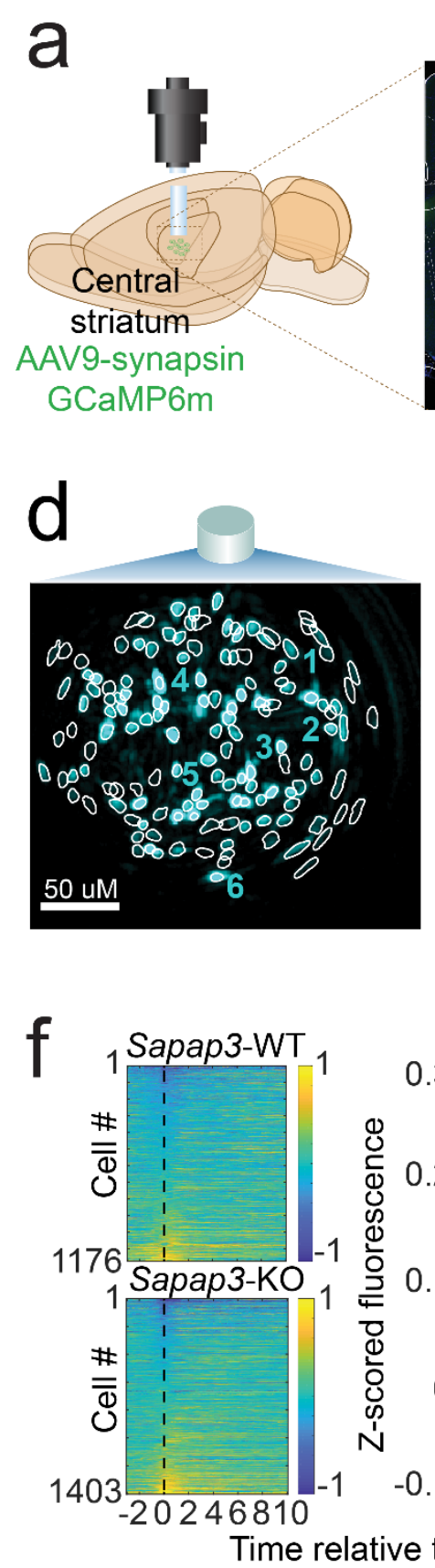

$\mathrm{h}$

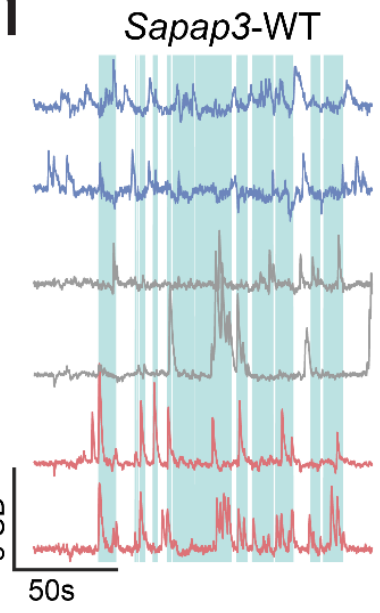

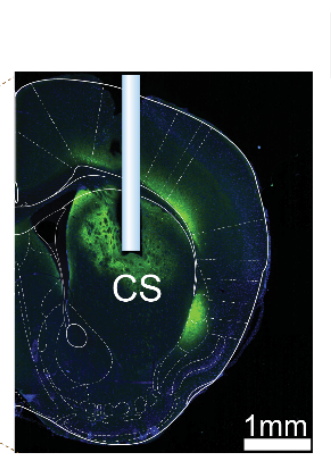
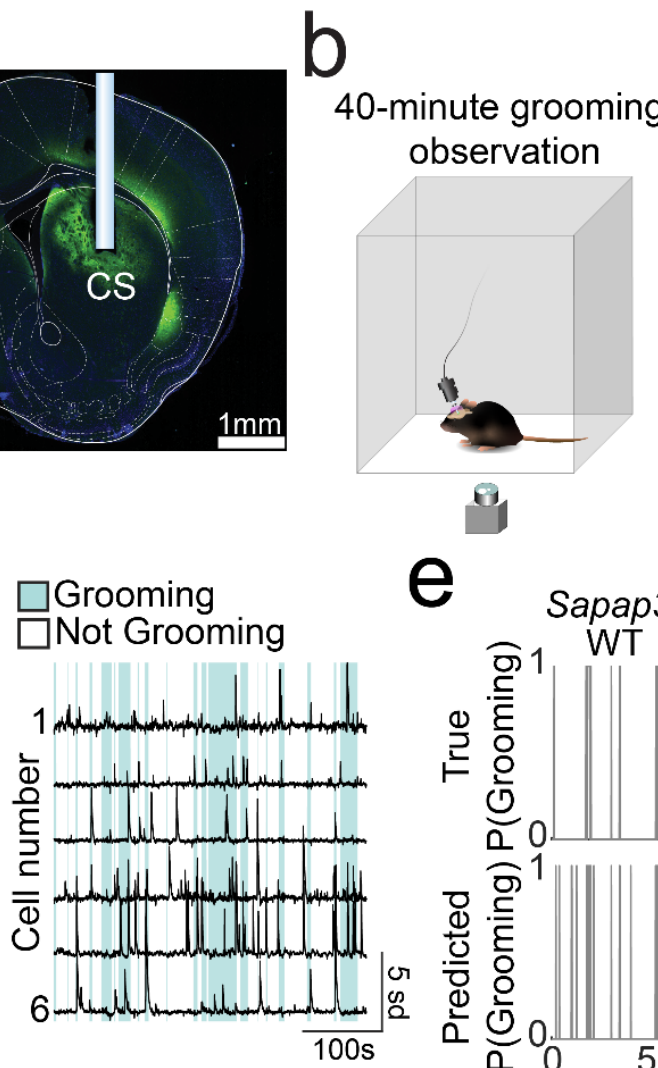

e

$c$
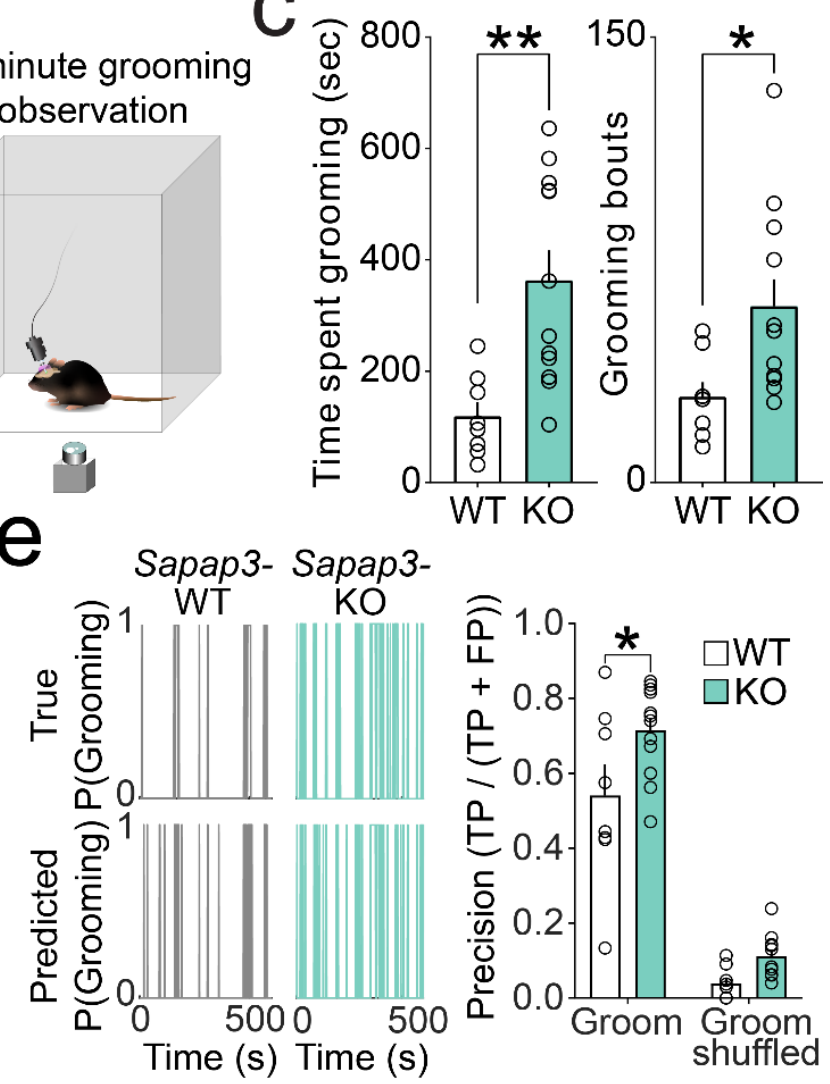
Figure 1. Central striatum is hyperactive in Sapap3-KO mice during grooming (a) Experimental design for imaging central striatal (CS) neurons (left) and representative histological image of GCaMP6m expression and lens placement in CS. (b) Schematic of behavioral apparatus. (c) Sapap3-KO mice $(n=11)$ spend significantly more time grooming (left; $t(18)=3.51, p=0.003$ ) and engage in significantly more grooming bouts (right; $t(18)=2.56, p=0.02$ ) than Sapap3-WT mice $(n=8)$. (d) Contour map of putative CS neurons overlayed on peak-to-noise ratio image (left). Individual traces of selected neurons in field of view (FOV) overlayed on top of grooming (blue) behavior (right). (e) Probability of grooming in a representative Sapap3-WT (left) and Sapap3-KO mouse (right). Top traces represent real grooming behavior; bottom represents grooming predicted via SVM decoder. SPN population decoding precision (true positives divided by true positives + false positives) is greater for grooming than for shuffled grooming behavior [main effect of behavior type; $F(1,17)=29.17, p<0.0001]$. Main effect of genotype $[F(1,17)=3.70, p=0.02]$. Interaction between genotype and behavior type $[F(1,17)=9.78, p=0.0061]$. Grooming decoding precision was improved in Sapap3-KOs (teal) relative to WTs (white) $[t(34)=2.98, p=0.01]$. (f) Trial averaged activity aligned to grooming start across all cells in Sapap3-WT (top) and Sapap3KO mice (bottom). Dotted line indicates groom start. CS neurons display elevated activity at grooming start in Sapap3KOs compared to Sapap3-WTs (all significant $t(2577) \geq 3.61, p \leq 0.00038$; thick black line in figure). (g) Calcium event rates are elevated during compulsive grooming in Sapap3-KOs $(t(17)=2.31, p=0.033)$. No difference in calcium event rates for all other times during the session $(t(17)=1.07, p=0.30)$. (h) Representative traces of cells classified as grooming-onset activated (red), grooming-onset inhibited (blue), and unaffected at grooming onset (grey) in a Sapap3-WT (left) and Sapap3-KO mouse (right). (i) Representative contour maps of individual CS neurons colored according to h. (j) Sapap3KO mice have a significantly greater percentage of CS neurons activated at grooming onset compared to Sapap3-WT mice $(t(17)=4.56, p=0.0003)$. No difference in percentage of grooming-onset inhibited cells between genotypes $(p>0.05)$. ${ }^{* * *}, p \leq 0.001,{ }^{* *} p \leq 0.01,{ }^{*} p \leq 0.05$. 

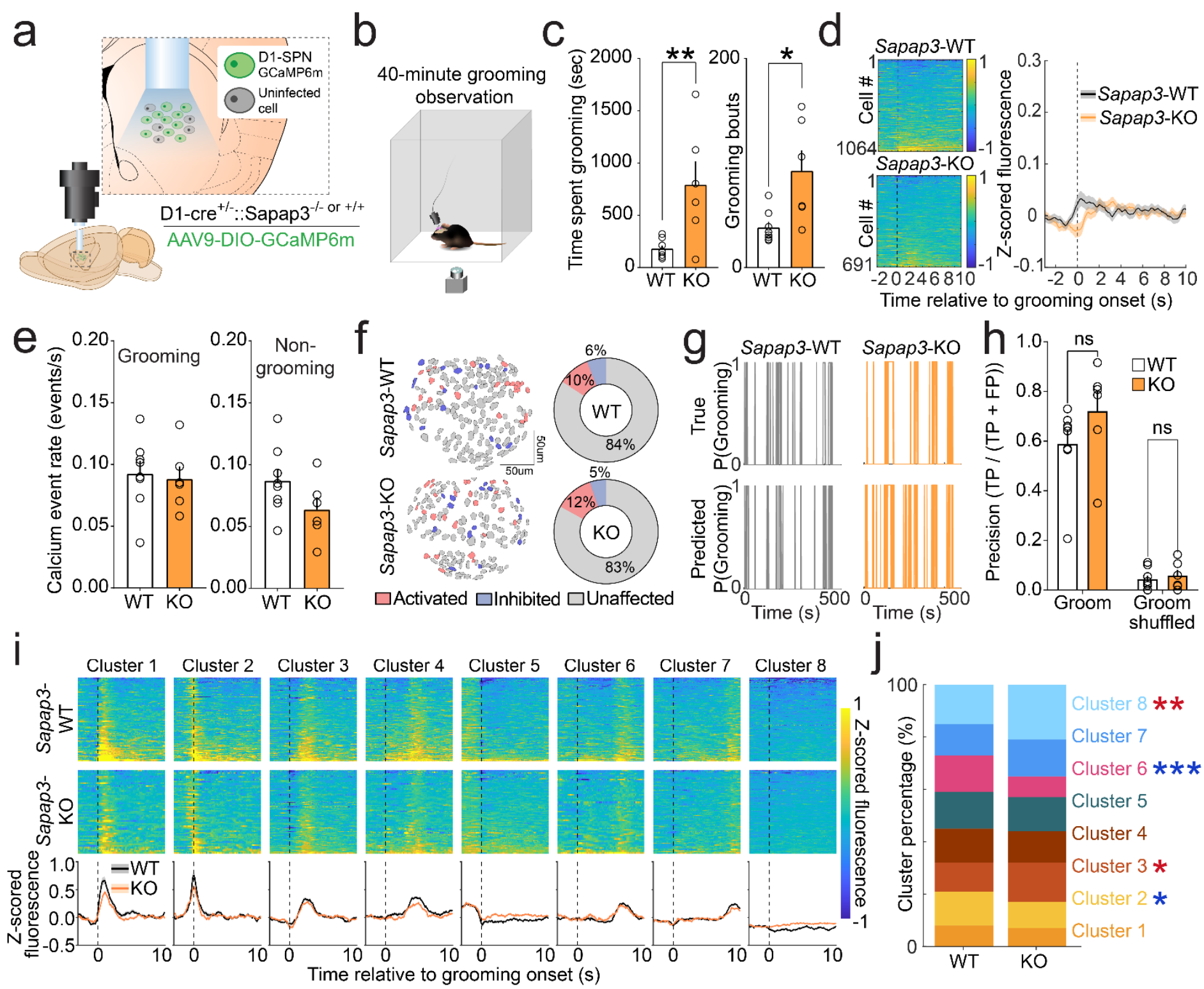

Figure 2. D1-SPNs are not hyperactive at grooming onset in Sapap3-KO mice. (a) Experimental design for selective imaging of D1-SPNs. (b) Schematic of behavioral apparatus. (c) Sapap3-KO ( $n=6)$ mice spend significantly more time grooming (left; $t(12)=3.3, p=0.009$ ) and engage in significantly more grooming bouts $(t(12)=3.0, p=0.01)$ than Sapap3-WT ( $n=8$ ) mice. (d) Trial averaged activity aligned to grooming start across all cells in Sapap3-WT (top; $n=1064$ ) and Sapap3KO mice (bottom; $n=691$ ). Dotted line indicates groom start. No significant difference between Sapap3-WTs and Sapap3KOs in overall grooming onset D1-SPN activity (all t(1753) $\geq 2.81, p \geq 0.00038$ ). (e) D1-SPN calcium event rates are not significantly different during grooming (left) and non-grooming (right) periods in Sapap3-KOs compared to WTs ( $p>0.05)$. (f) Contour map of D1-SPNs colored according to their activity (red=activated, blue=inhibited, grey=unaffected) at onset of grooming (left). Average percentage of activated, inhibited, and unaffected D1-SPNs in WT (top) and Sapap3-KO mice (bottom). (g) Probability of grooming in a representative Sapap3-WT (left) and Sapap3-KO mouse (right). Top traces represent real grooming behavior; bottom represents grooming predicted via SVM decoder based on D1-SPN population 
activity. (h) D1-SPN population decoding precision (true positives / true positives + false positives) is greater for grooming than for shuffled grooming (main effect of behavior type; $F(1,12)=132.9, p<0.0001$ ). No main effect of genotype $(F(1,12)=2.29, p=0.16)$ or interaction between genotype and behavior type was observed $(F(1,12)=1.26, p=0.28)$. (i) Functional clustering of trial averaged grooming-onset activity in D1-SPNs in WT (top) and Sapap3-KO mice (middle) represented as a heatmap. Functional clustering identified 8 distinct functional clusters (bottom). Mean grooming-onset activity of each cluster in WT (black) and Sapap3-KOs (orange) (j) Compared to WTs, Sapap3-KOs have a greater proportion of Cluster $3\left(X^{2}(1)=4.77, p=0.03\right)$ and Cluster $8\left(X^{2}(1)=8.76, p=0.003\right)$ D1-SPNs, a significantly lower proportion of Cluster $2\left(X^{2}(1)=3.62, p=0.05\right)$ and Cluster $6\left(X^{2}(1)=16.53, p=0.0001\right) \mathrm{D} 1$-SPNs, and no changes in Clusters $1,4,5$, and $7 .{ }^{* * *}, p \leq 0.001,{ }^{* *} p \leq 0.01,{ }^{*} p \leq 0.05$. 

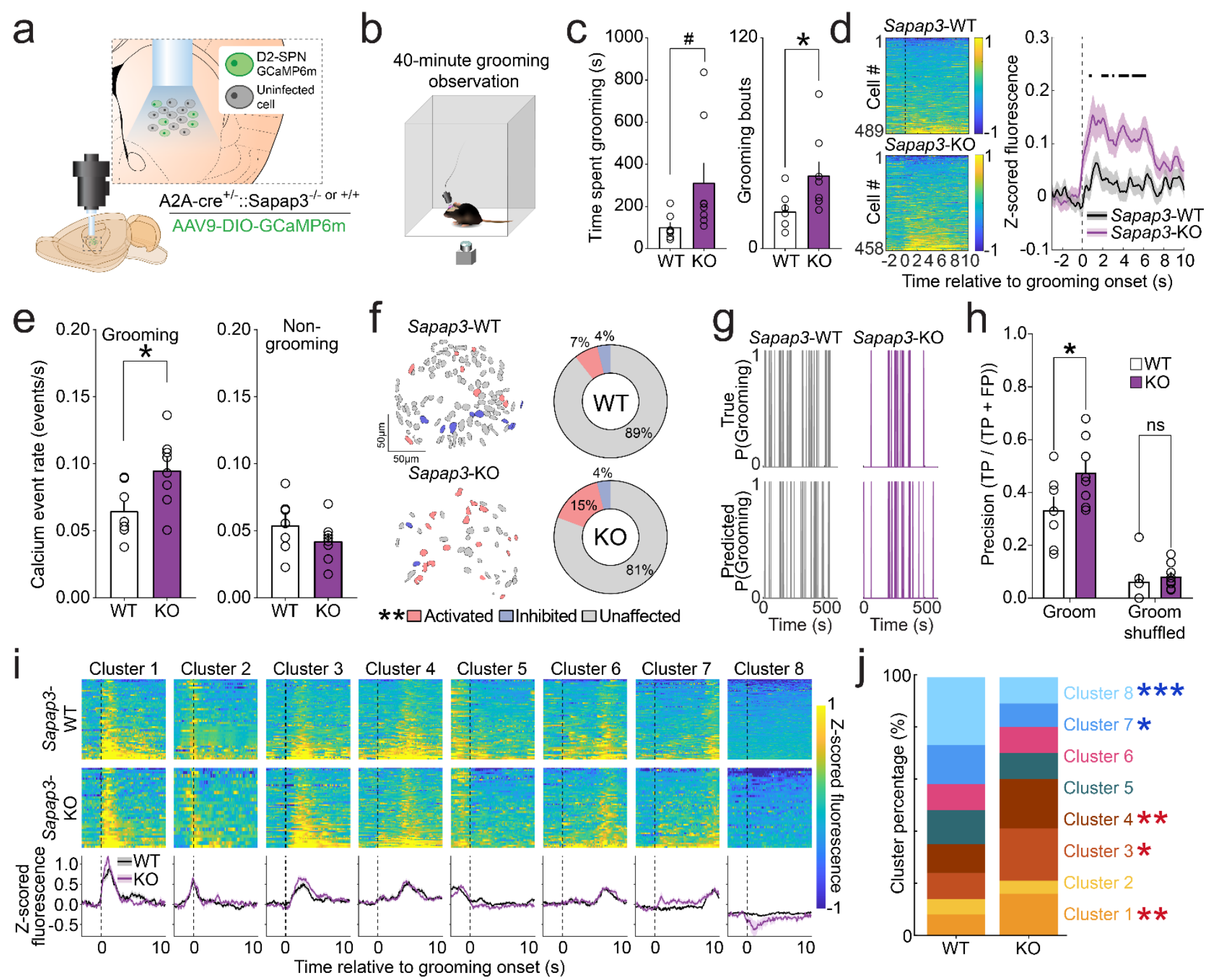

Figure 3. D2-SPNs are hyperactive at grooming onset in Sapap3-KO mice. (a) Experimental design for selective

imaging of D2-SPNs. (b) Schematic of behavioral apparatus. (c) Sapap3-KO ( $n=8)$ mice spend more time grooming (left; $t(13)=2.0, p=0.06)$ and engage in significantly more grooming bouts (right; $t(13)=2.2, p=0.046)$ than Sapap3-WT ( $n=7$ ) mice. (d) Trial averaged activity aligned to grooming start across all cells in Sapap3-WT (top; $n=489$ ) and Sapap3-KO mice (bottom; $n=458$ ). Dotted line indicates groom start. Groom-start aligned D2-SPN activity is significantly elevated in Sapap3-KOs compared to WTs (all significant $t(945) \geq \pm 3.58, p \leq 0.00038$; thick black lines in figure). (e) D2-SPN calcium event rates are elevated during grooming (left; $t(13)=2.5, p=0.028$ ) and unchanged during non-grooming (right; $p>0.05$ ) periods in Sapap3-KOs compared to WTs. (f) Contour map of D2-SPNs colored according to their activity (red=activated, blue=inhibited, grey=unaffected) at the onset of grooming (left). Sapap3-KOs have a greater proportion of groom-onset activated D2-SPNs than WTs $(t(13)=1.99, p=0.034)$, with no changes in the proportion of inhibited or unaffected neurons $(p>0.05)$. (g) Probability of grooming in a representative Sapap3-WT (left) and Sapap3-KO mouse (right). Top traces 
represent real grooming behavior; bottom represents grooming predicted via SVM decoder based on D2-SPN population activity. (h) D2-SPN population decoding precision (true positives divided by true positives + false positives) is greater for grooming than for shuffled grooming [main effect of behavior type; $F(1,13)=98.62, p<0.0001$ ]. A trend toward a significant main effect of genotype $[F(1,13)=4.45, p=0.055]$ and significant interaction between genotype and behavior type was observed $[(F(1,13)=4.78, p=0.048]$. (i) Functional clustering of trial averaged grooming-onset activity in D2-SPNs in WT (top) and Sapap3-KO mice (middle) represented as a heatmap. Functional clustering identified 8 distinct functional clusters (bottom). Mean grooming-onset activity of each cluster in WT (black) and Sapap3-KO mice (purple). (j) Compared to WTs, Sapap3-KO have a greater proportion of Cluster $1\left(X^{2}(1)=6.75, p=0.01\right)$, Cluster $3\left(X^{2}(1)=5.076\right.$, $p=0.024)$ and Cluster $4\left(X^{2}(1)=7.65, p=0.006\right)$ D2-SPNs, a significantly lower proportion of Cluster $7\left(X^{2}(1)=5.52, p=0.02\right)$ and Cluster $8\left(X^{2}(1)=27.09, p<0.0001\right)$ D2-SPNs, and no changes in Clusters 2, 5 , and 6. ${ }^{* * *}, p \leq 0.001,{ }^{* *} p \leq 0.01,{ }^{*} p \leq 0.05$, $\# p \leq 0.1$. 

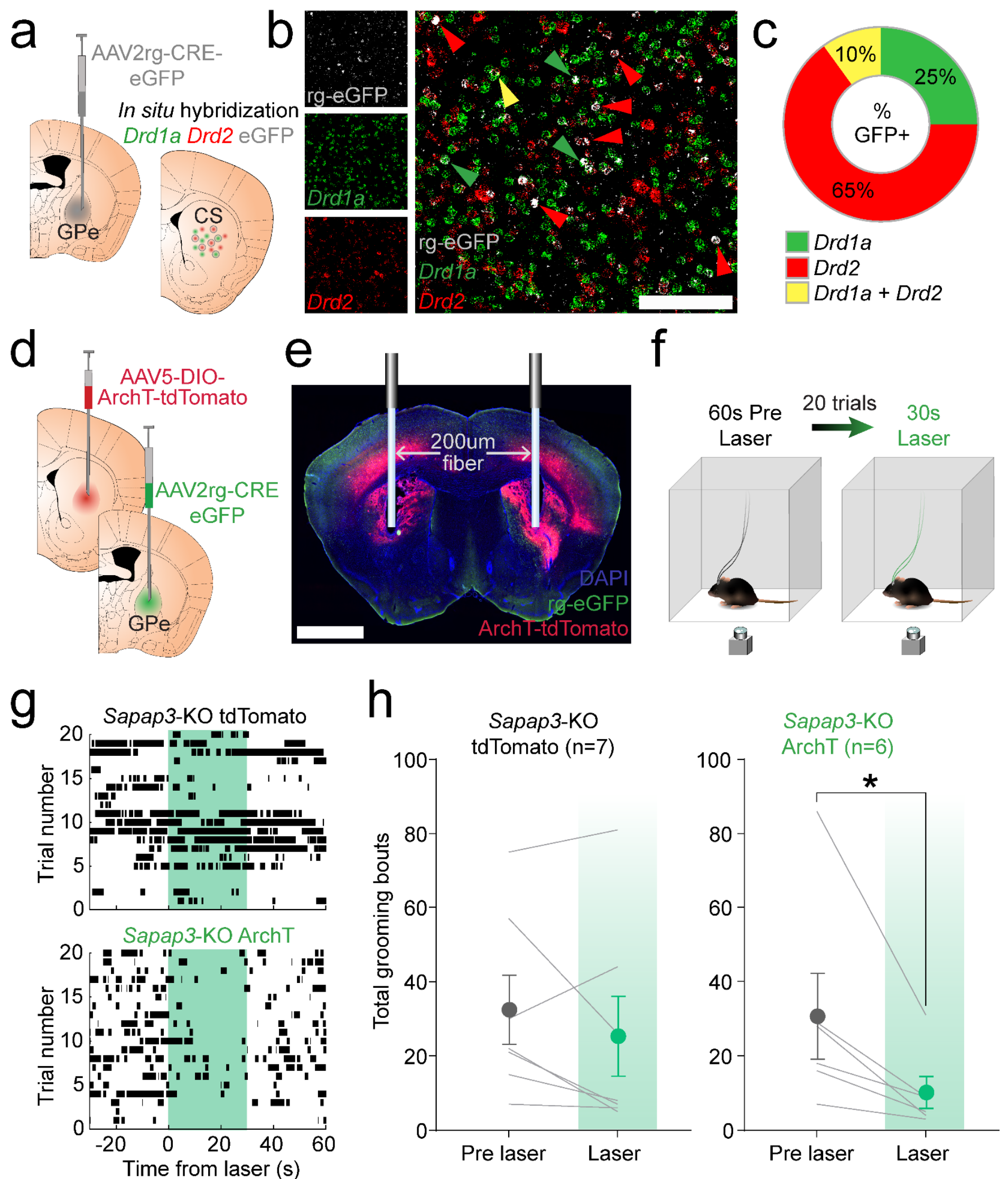

Figure 4. Inhibition of striatopallidal iSPNs reduces compulsive grooming behavior. (a) Schematic of strategy for assessing iSPN expression of dopamine D1 and D2 receptors. Retrograde AAV2-CRE-eGFP was injected into globus 
pallidus external segment (GPe) and in situ hybridization performed 10 weeks later in CS for eGFP, Drd1a and Drd2. (b) In situ hybridization image for retrograde eGFP (grey), Drd1a (green), and Drd2 (red). Arrows indicate eGFP+ neurons overlapping with Drd1a (green), Drd2 (red), or both Drd1a and Drd2 (yellow). Scale bar = 100um. (c) Average percentage of eGFP+ neurons expressing Drd1a (25\%), Drd2 (65\%), or both (10\%) in CS ( $n=5$ CS slices from 5 Sapap3-WT mice). (d) Schematic for inhibition of iSPNs. Retrograde AAV2-CRE-eGFP was injected bilaterally into GPe and AAV5-DIOArchT-tdTomato or AAV5-DIO-tdTomato was injected bilaterally into CS of WT and KO mice. (e) 200um optic fibers were placed bilaterally into CS; scale bar $=2 \mathrm{~mm}$. (f) Mice received 20 trials of $30 \mathrm{~s}$ of $532 \mathrm{~nm}$ laser stimulation followed by 60 s of no laser, while behavior was recorded from a bottom view camera. (g) Raster plots of grooming bouts (black tic marks) during laser trials in representative Sapap3-KO tdTomato (top) and ArchT (bottom) mice. Green shading indicates duration of laser illumination (30s). (h) No effect of laser illumination on number of grooming bouts in Sapap3-KOs expressing tdTomato control virus $(n=7 ; t(6)=1.25, p=0.26)$. In Sapap3-KO mice expressing ArchT in iSPNs, laser illumination significantly reduced number of grooming bouts $(n=6 ; t(5)=2.73, p=0.042)$. ${ }^{*} p \leq 0.05$. 

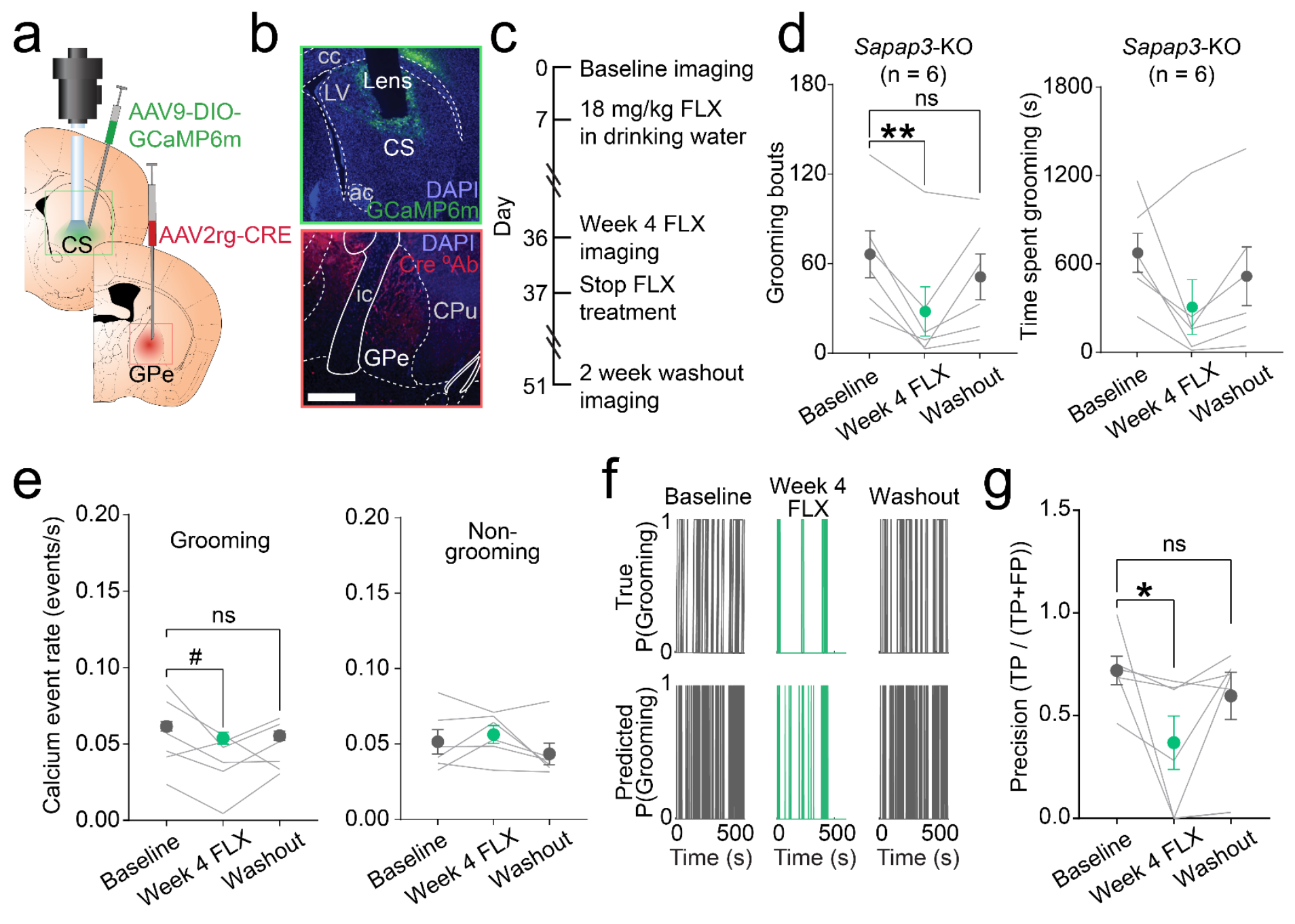

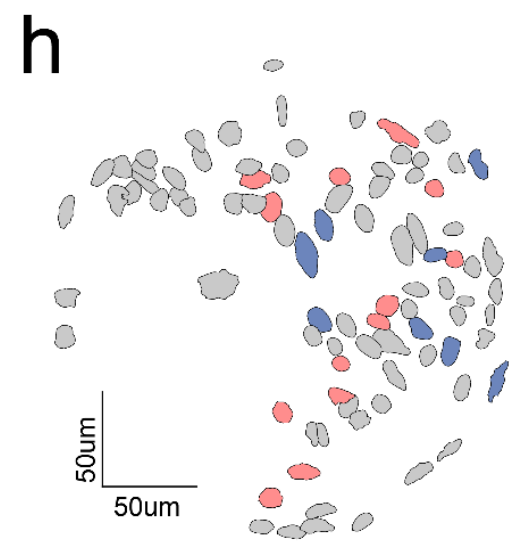

$\square$ Activated $\square$ Inhibited $\square$ Unaffected
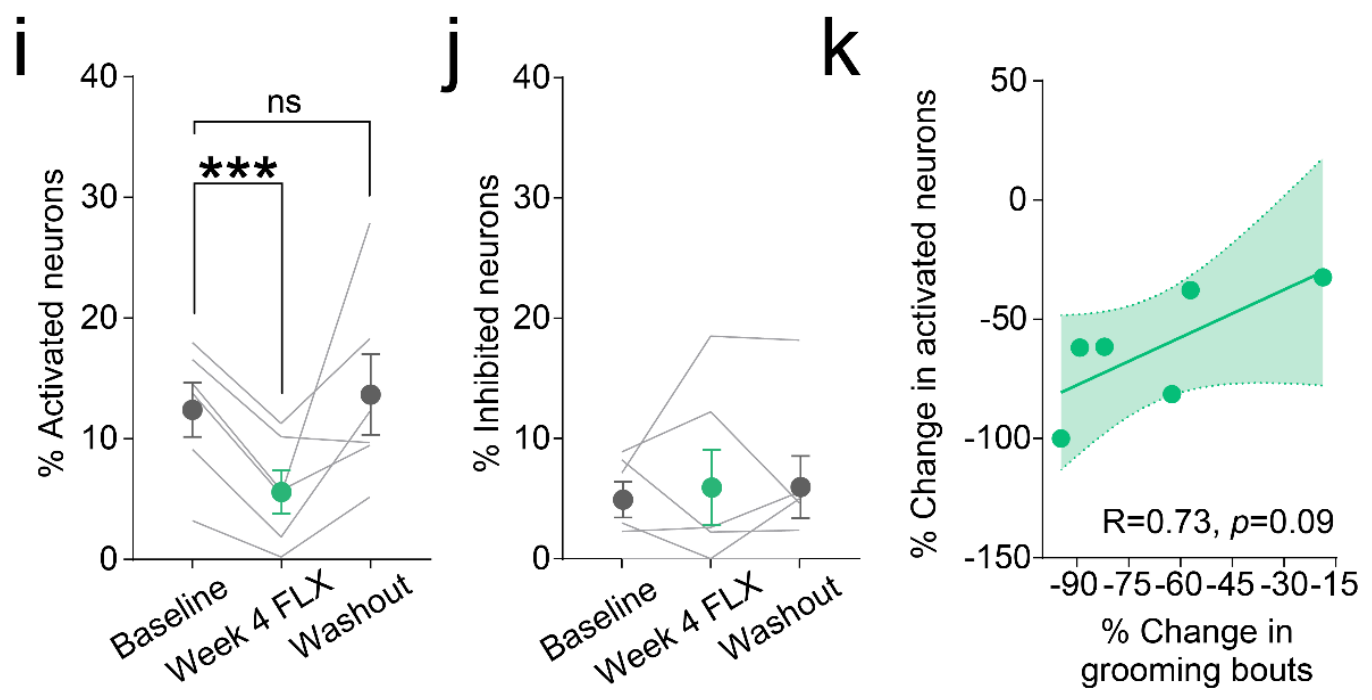

Figure 5. Fluoxetine reduces compulsive grooming behavior and iSPN hyperactivity. (a) Schematic of strategy for assessing effect of fluoxetine on iSPN activity during compulsive grooming. Retrograde AAV2-CRE was injected unilaterally into GPe and AAV9-DIO-GCaMP6m into ipsilateral CS followed by GRIN lens placement. (b) (top) GCaMP6m expression in iSPNs and GRIN lens track in CS. (bottom) Immunohistochemical stain for Cre-recombinase (red) in GPe. Scale bar $=1 \mathrm{~mm}$. (c) Timeline for experiments evaluating effect of fluoxetine on compulsive grooming behavior and iSPN 
activity. (d) (left) Fluoxetine significantly reduced number of grooming bouts in Sapap3-KOs ( $n=6$; RM ANOVA main effect of treatment $[F(2,5)=7.08, p=0.0214)$; Baseline vs. Week $4 \mathrm{FLX}$ post-hoc $(t(5)=5.21, p=0.004)$; other comparisons $p>0.05]$. (right) No effect of treatment on time spent grooming [RM ANOVA main effect of treatment $(F(2,5)=3.5, p=0.11)]$. (e) (left) Trend toward reduction in grooming-associated calcium event rate following fluoxetine (RM ANOVA main effect of treatment $(F(2,5)=3.72, p=0.037)$ post-hoc $(t(5)=2.60, p=0.082))$. (right) No effect of fluoxetine on iSPN event rate during non-grooming time (RM ANOVA main effect of treatment $(F(2,5)=2.43, p=0.14)$ ). (f) (top) True grooming behavior during baseline, week 4 fluoxetine, and washout sessions in representative animal. (bottom) SVM-predicted grooming based on iSPN population activity across days. (g) Precision of SVM for classification of grooming behavior during baseline, week 4 fluoxetine, and washout sessions (baseline vs. week 4 fluoxetine, $t(15)=2.3, p=0.04$ ). (h) Contour map of striatopallidal iSPNs from representative KO colored according to baseline activity at grooming onset ( $\mathrm{red}=\mathrm{activated}$, blue $=$ inhibited, grey $=$ unaffected). (i) Fluoxetine reduces percentage of grooming-onset activated striatopallidal iSPNs [RM ANOVA main effect of treatment $(F(2,5)=5.52, p=0.05)$; Baseline vs. Week $4 \mathrm{FLX}$ post-hoc $(t(5)=8.01, p=0.002)]$. (j) No effect of treatment on percentage of grooming-onset inhibited striatopallidal iSPNs [RM ANOVA main effect of treatment $(F(2,5)=0.13, p=0.84)]$. (k) Positive correlation between the percent change in grooming bouts and percent change in activated neurons following fluoxetine treatment in $\mathrm{KO}$ mice $(R=0.73, p=0.09) .{ }^{* * *}, p \leq 0.001,{ }^{* *} p \leq 0.01,{ }^{*} p \leq 0.05, \# p \leq 0.1$. $\mathrm{cc}=$ corpus callosum, $\mathrm{LV}=$ lateral ventricle, ac=anterior commissure, ic=internal capsule, $\mathrm{CPu}$, caudate/putamen. 


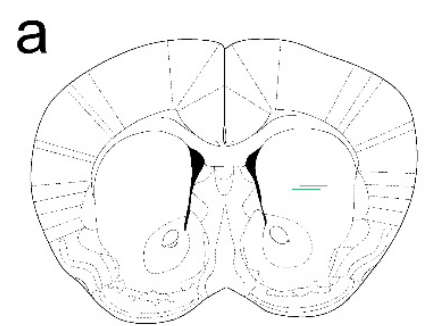

Bregma $1.10 \mathrm{~mm}$

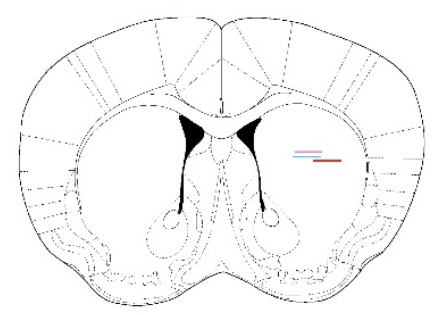

Bregma $0.86 \mathrm{~mm}$

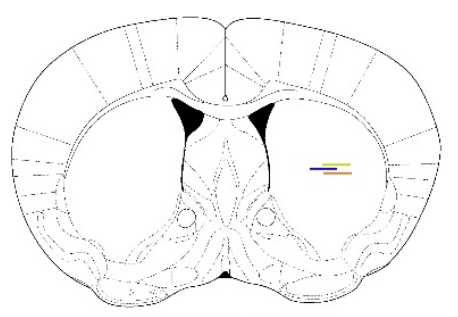

Bregma $0.50 \mathrm{~mm}$

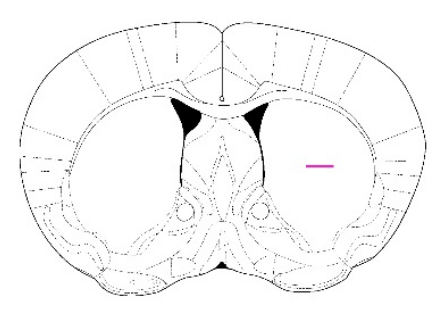

Bregma 0.62 mm

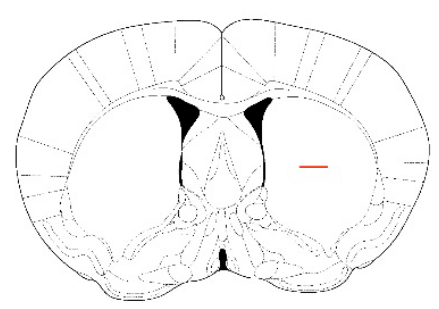

Bregma 0.38 mm

-3220
-3320

$-776$

$-3319$

780

$-3231$

$-3328$

$-3334$

$-522$

$-3321$
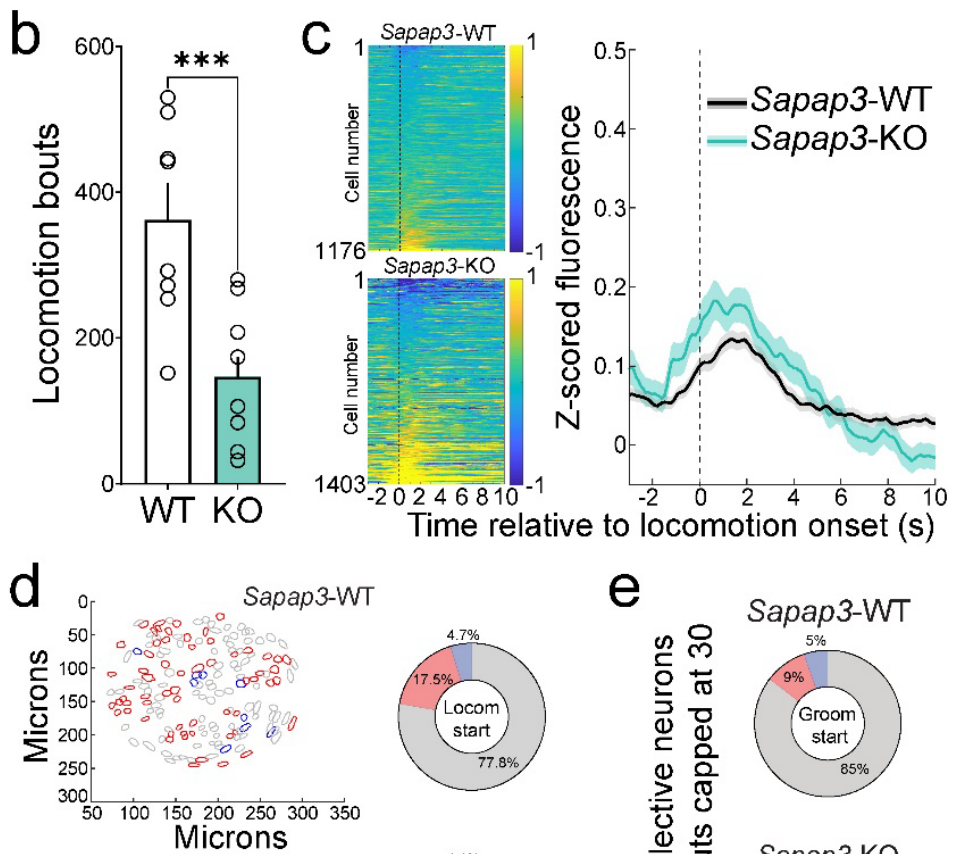

e
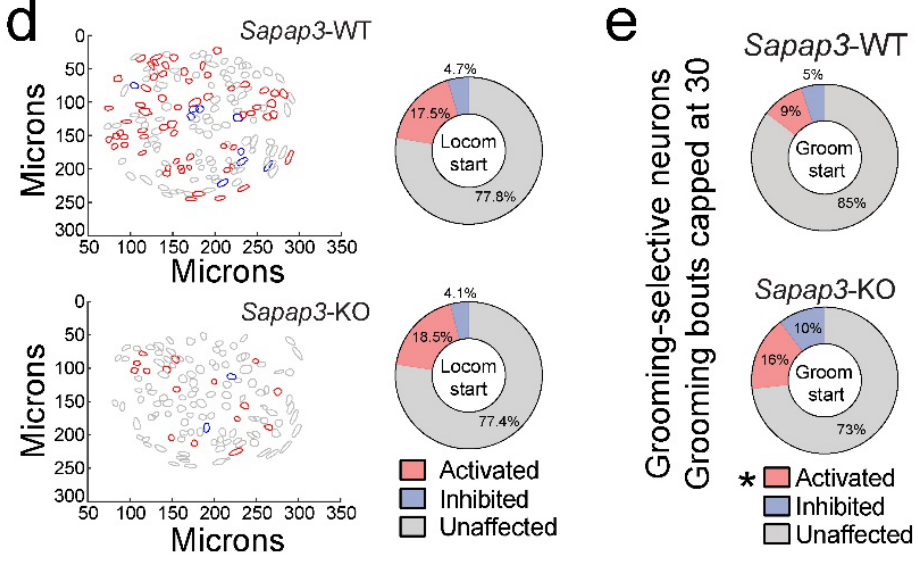

\section{Extended Data Figure 1}

Extended Data Figure 1. Extended data from CS calcium imaging. (a) Lens placements in CS for subset of mice included in Figure 1. Individual horizontal lines indicate bottom of GRIN lens for a single mouse. (b) Sapap3-KOs engage in fewer bouts of locomotion relative to WT littermates $(t(17)=4.21, p=0.0006)$. (c) Locomotion-start aligned calcium fluorescence averaged across trials from CS SPNs in WT (top) and KO (bottom) mice. Locomotion-start aligned calcium activity is not significantly different in WT and KO mice (right, p $\geq 0.00038$ ). (d) Contour maps of same WT and KO mouse from Figure 1i, now colored according to locomotion-start activity. No significant differences observed in proportion of locomotion-start activated (red), inhibited (blue), or unaffected (grey) SPNs in WTs and KOs (all p>0.05). (e) Proportion of grooming-selective neurons identified in WTs and KOs when grooming bouts were capped to mean number of WT grooming bouts (30 bouts). Compared to WTs, KOs still had significantly greater proportions of grooming-onset activated neurons $(t(17)=2.84, p=0.01)$. No differences in grooming-onset inhibited neurons were identified $(p>0.05)$. 

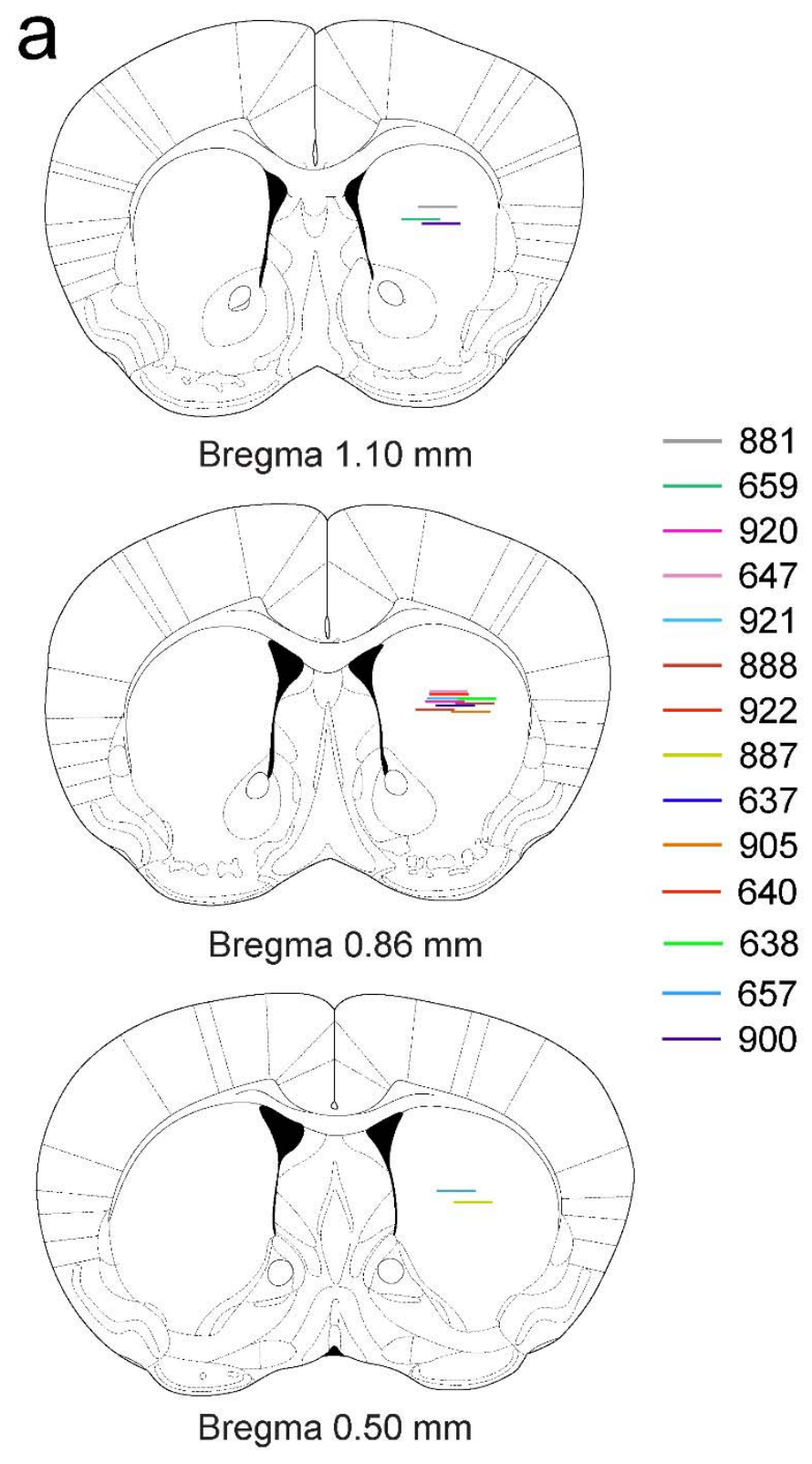

\section{Extended Data Figure 2}

Extended Data Figure 2. Lens placement for D1-Cre / Sapap3-KO and -WT imaging. (a) Histological verification of GRIN lens placements in CS. Individual horizontal lines indicate bottom of GRIN lens for a single mouse. 
a
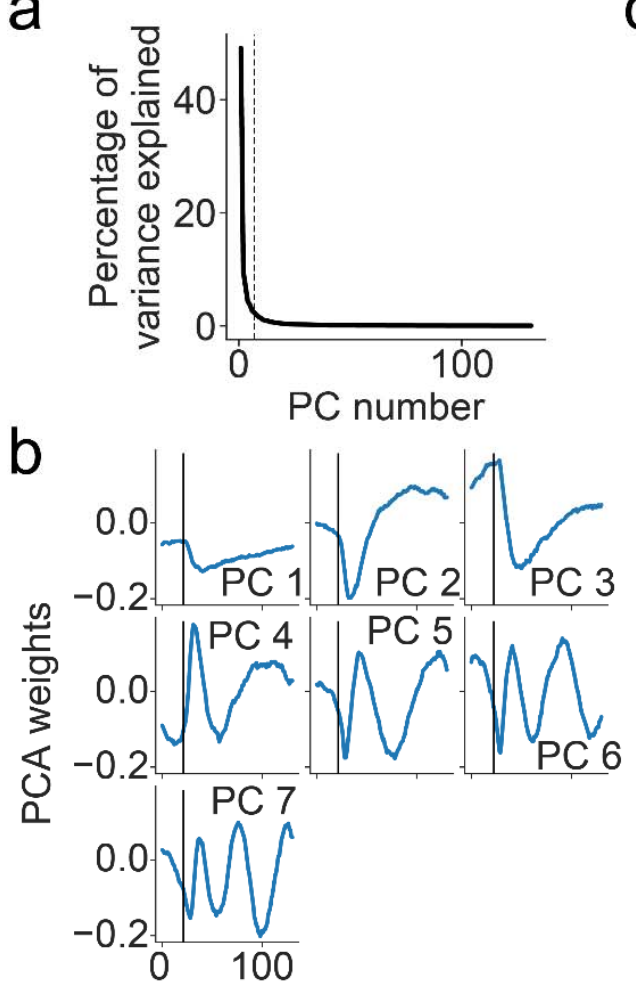

C
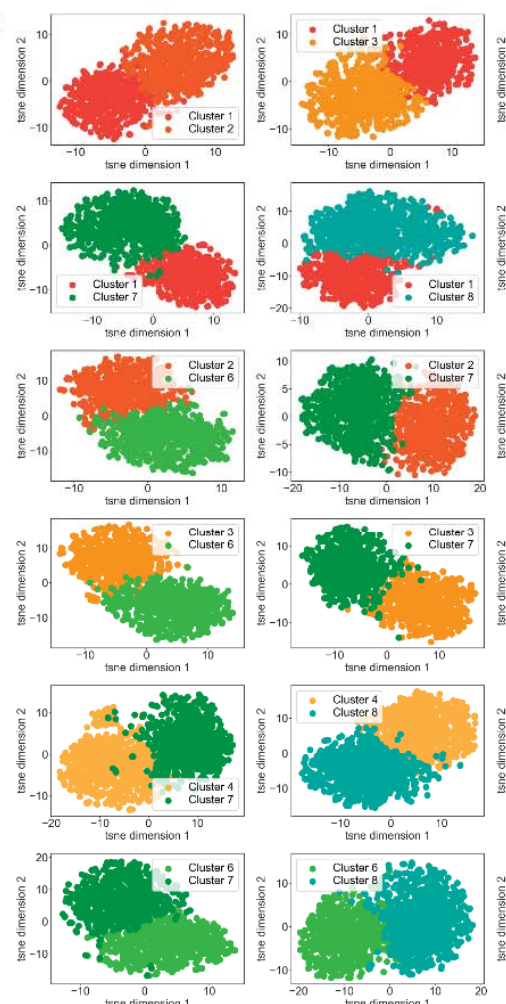
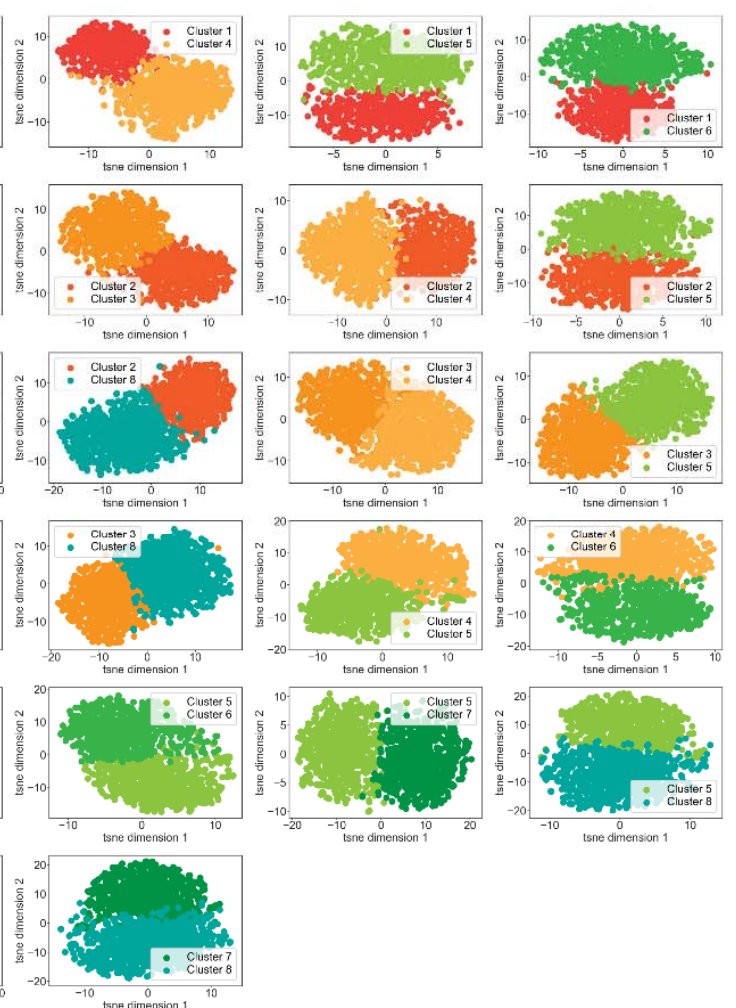

Extended Data Figure 3

Extended Data Figure 3. Hierarchical clustering analysis of central striatal SPNs. (a) Scree plot of percentage of variance explained per principal component; dotted line indicates number of principal components retained (7). (b) Plots of individual retained components. (c) Cluster separation in activity space. Neurons belonging to each pair of clusters are shown in a 2D t-distributed Stochastic Neighbor Embedding (t-SNE) plot to show that each cluster is separable from other clusters. Perplexity was set to 100 for this visualization. 


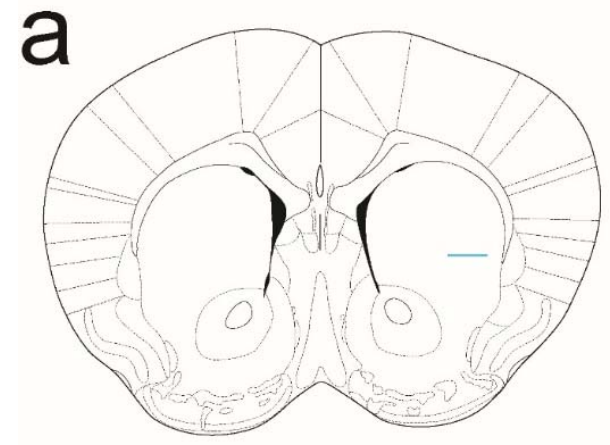

Bregma $1.18 \mathrm{~mm}$

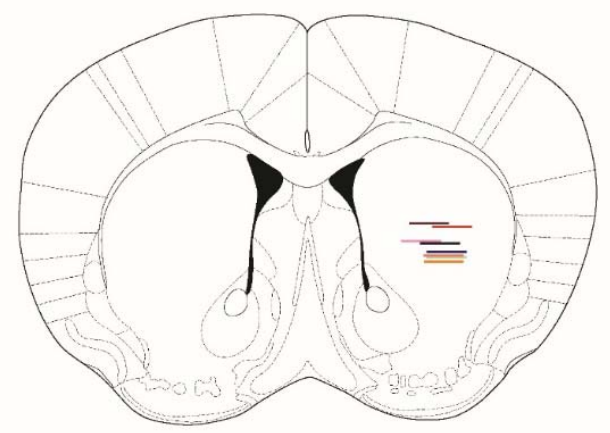

Bregma $0.86 \mathrm{~mm}$

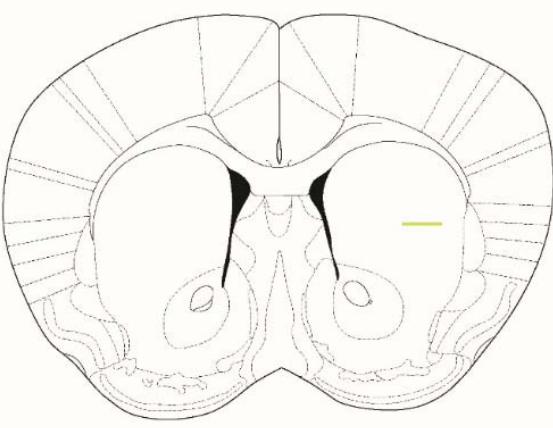

Bregma $1.10 \mathrm{~mm}$

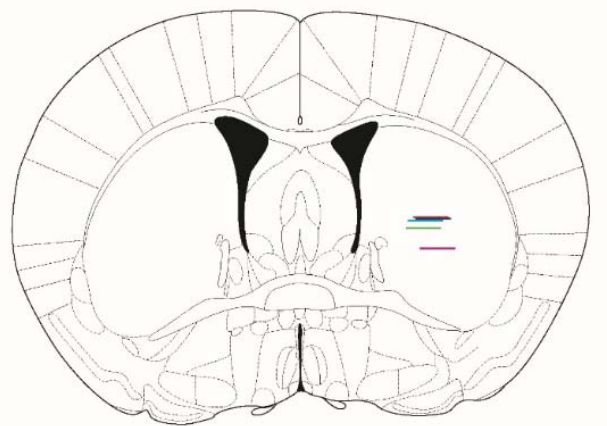

Bregma $0.14 \mathrm{~mm}$
$-160$

106

119

126

278

54

114

$-128$

225

157

$-125$

149

124

176

$-246$

\section{Extended Data Figure 4}

Extended Data Figure 4. Lens placements for A2a-Cre ${ }^{+/-}$/ Sapap3-KO and -WT imaging. (a) Histological verification of GRIN lens placements in CS. Individual horizontal lines indicate bottom of GRIN lens for a single mouse. 


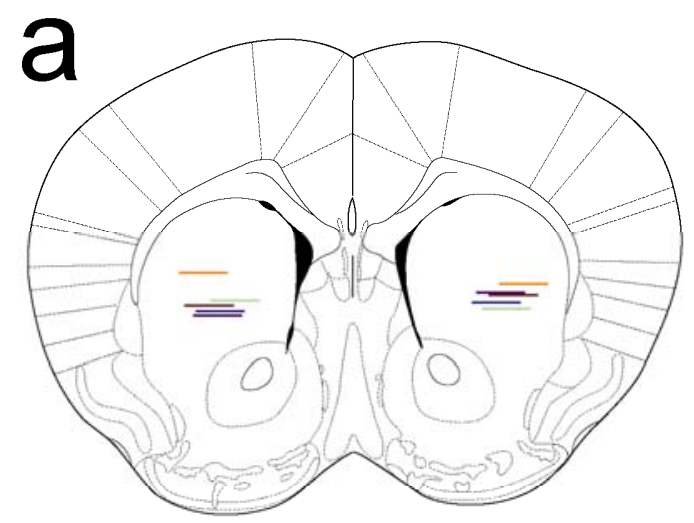

Bregma $1.18 \mathrm{~mm}$

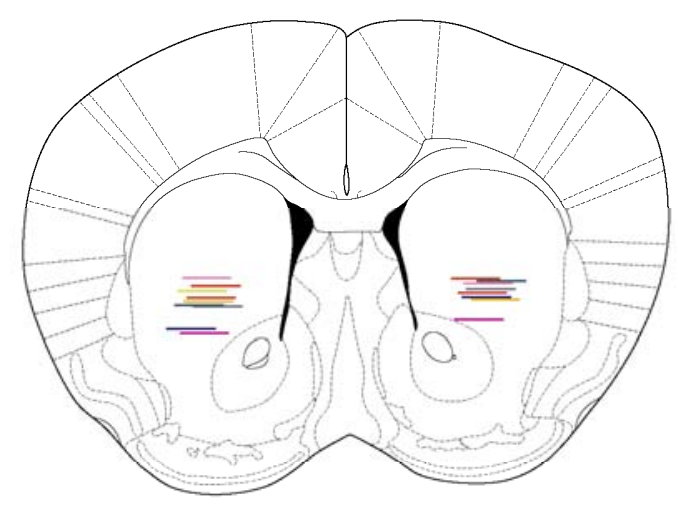

Bregma $1.10 \mathrm{~mm}$
$-6201$

$-6220$

6248

$-6254$

$-6259$

$-6261$

6272

6279

$-6292$

$-6294$

$-6295$

$-6297$

$-6299$

6218

$-6221$

6238

$-6251$

6260

$-6267$

$-6296$

Bregma $0.86 \mathrm{~mm}$

Extended Data Figure 5

Extended Data Figure 5. Retro-GPe fiberoptic implant histology. (a) Histological verification of bilateral optic fiber placements in CS of KO (13 included, 5 excluded) and WT (7 included, 1 excluded) mice. Individual horizontal lines indicate bottom of optic fiber for a single mouse. 

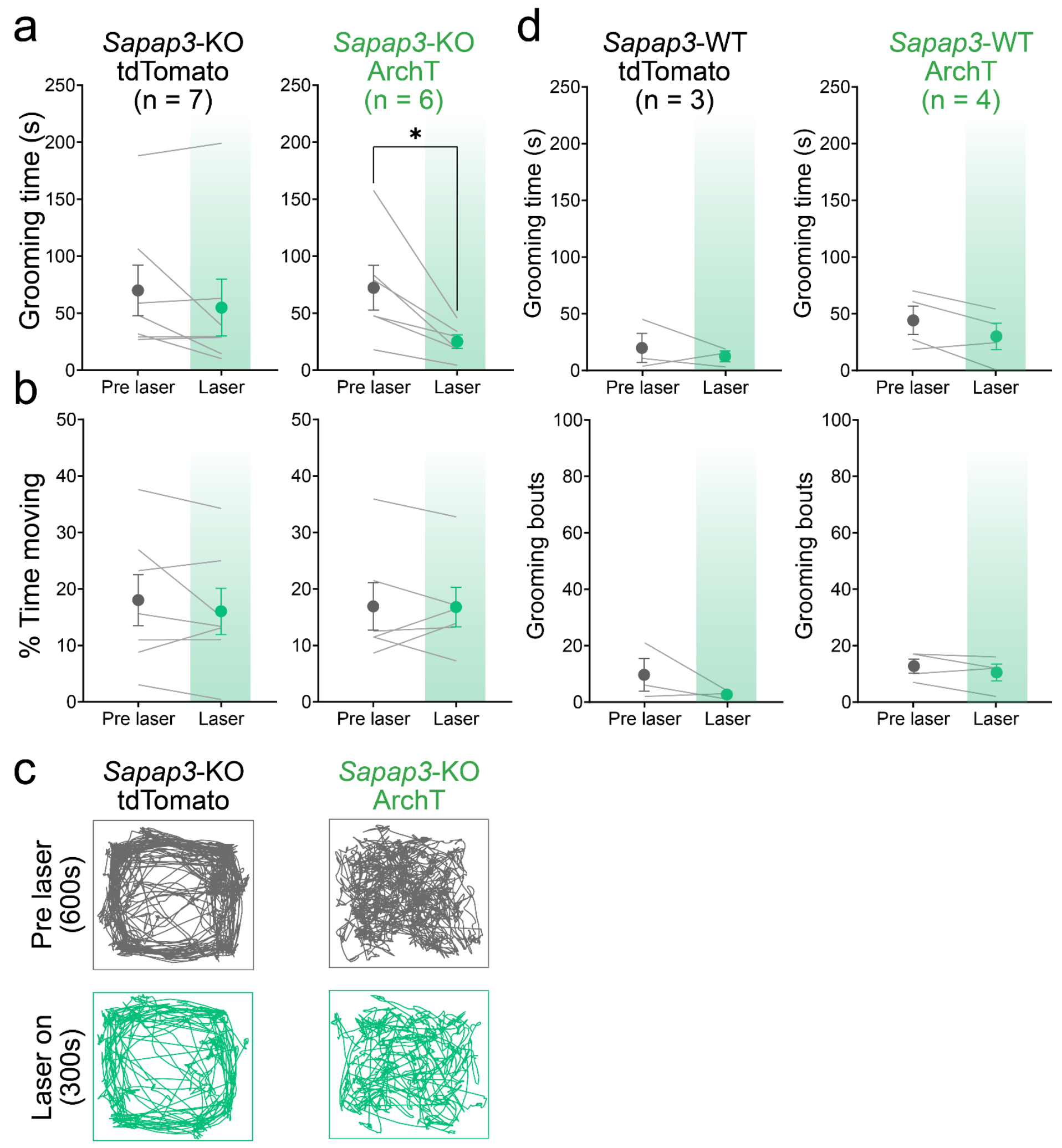

\section{Extended Data Figure 6}

Extended Data Figure 6. Inhibition of striatopallidal SPNs. (a) No effect of laser illumination on time spent grooming in tdTomato-expressing KO mice (left; $p>0.05$ ). Significant reduction in time spent grooming during laser illumination in ArchT-expressing KO mice $(t(5)=3.13, p=0.03)$. (b) No effect of laser illumination on percentage of total time spent moving 
compared to pre-laser time in either tdTomato- (left) or ArchT-expressing (right) KO mice (all $p>0.05$ ). (c) Locomotion traces of pre-laser (top) and laser on (bottom) periods across an entire session in example tdTomato- (left) and ArchTexpressing (right) KO mice. (d) No effect of laser illumination on time spent grooming (top) or number of grooming bouts (bottom) in tdTomato- (left) or ArchT-expressing (right) WT littermates (all $p>0.05$ ). 

bioRxiv reprint dol: https://doi.org/10.1101/2022.02.17.480966; this version posted February 18,2022 . The copyright holder for this preprint
(which was not certified by peer review) is the author/funder, who has granted bioRxiv a license to display the preprint in perpetuity. It is made

a
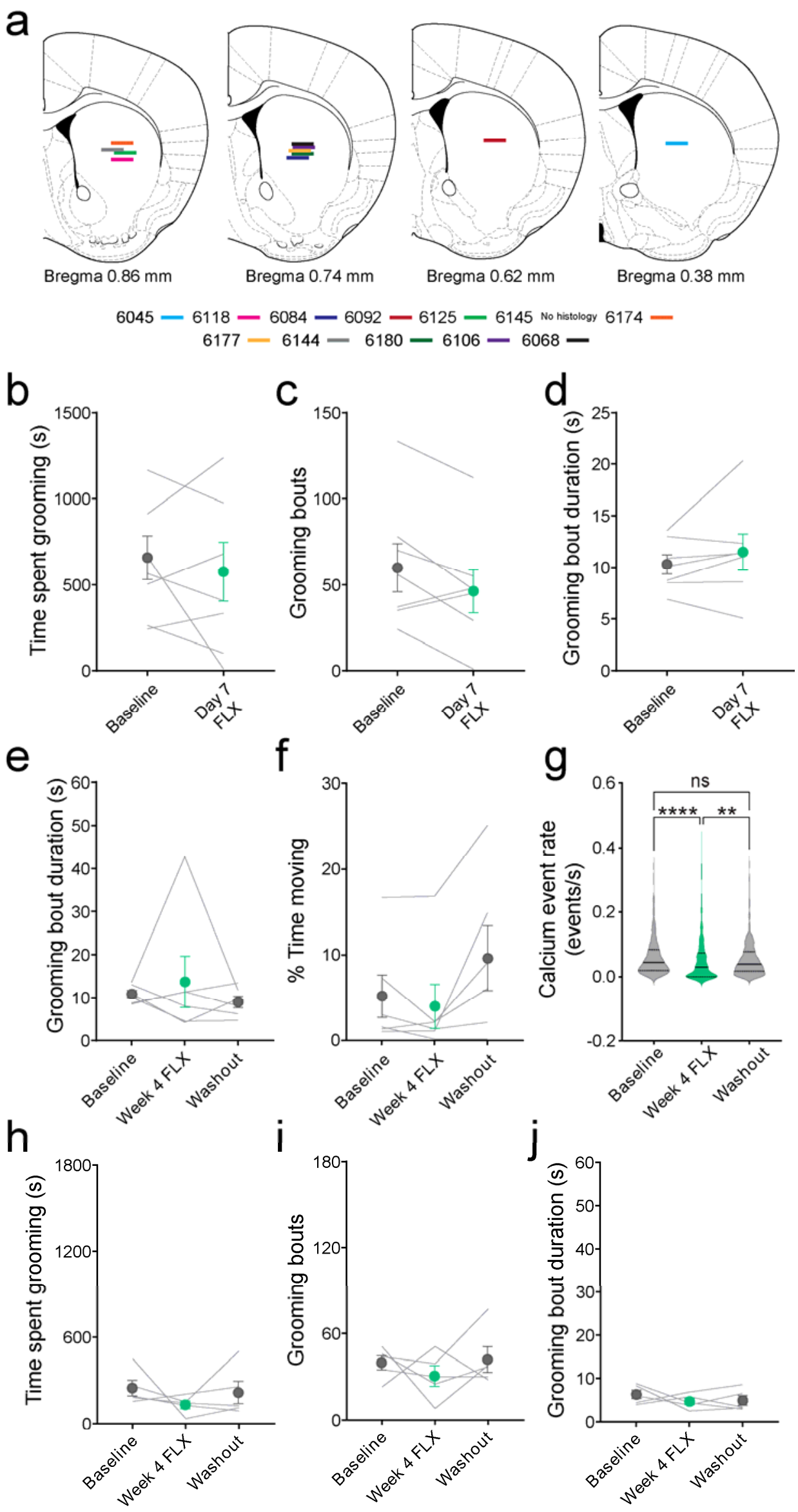

Extended Data Figure 7 
Extended Data Figure 7. Fluoxetine administration during imaging of striatopallidal SPNs. (a) Histological verification of GRIN lens placements in CS of WT and KO mice. (b) 7 days of intraperitoneal fluoxetine (FLX; $5 \mathrm{mg} / \mathrm{kg})$ treatment did not affect time spent grooming, (c) number of grooming bouts, or (d) grooming bout duration in Sapap3-KOs (all p>0.05). (e) Chronic fluoxetine treatment in drinking water did not affect grooming bout duration or (f) percentage of time spent moving during a 40 minute session (all $p>0.05$ ). (g) Effect of chronic fluoxetine on calcium event rate of individual striatopallidal SPNs (Kruskal-Wallis ANOVA, $H=23.29, p<0.0001$ ). Fluoxetine significantly reduced striatopallidal calcium event rate compared to baseline (Dunn's multiple comparison test, $Z=4.77, p<0.0001$ ) and washout periods (Dunn's multiple comparison test, $Z=3.12, p=0.01$ ). No effect of chronic fluoxetine on (h) time spent grooming, (i) number of grooming bouts, or (j) grooming bout duration in WT littermates (all $p>0.05$ ). 


\section{References}

1. Figee, M. et al. Deep brain stimulation restores frontostriatal network activity in obsessivecompulsive disorder. Nature neuroscience 16, 386-7 (2013).

2. Harrison, B. J. et al. Altered corticostriatal functional connectivity in obsessive-compulsive disorder. Arch Gen Psychiatry 66, 1189-200 (2009).

3. Menzies, L. et al. Integrating evidence from neuroimaging and neuropsychological studies of obsessive-compulsive disorder: the orbitofronto-striatal model revisited. Neuroscience and biobehavioral reviews 32, 525-549 (2008).

4. Rauch, S. L., Savage, C. R., Alpert, N. M., Fischman, A. J. \& Jenike, M. A. The functional neuroanatomy of anxiety: a study of three disorders using positron emission tomography and symptom provocation. Biol Psychiatry 42, 446-52 (1997).

5. Saxena, S. \& Rauch, S. L. Functional neuroimaging and the neuroanatomy of obsessivecompulsive disorder. The Psychiatric clinics of North America 23, 563-86 (2000).

6. Graybiel, A. M. \& Rauch, S. L. Toward a neurobiology of obsessive-compulsive disorder. Neuron 28, 343-347 (2000).

7. Rotge, J. Y. et al. Meta-analysis of brain volume changes in obsessive-compulsive disorder. Biol Psychiatry 65, 75-83 (2009).

8. Maia, T. V., Cooney, R. E. \& Peterson, B. S. The neural bases of obsessive-compulsive disorder in children and adults. Development and psychopathology 20, 1251-83 (2008).

9. Pauls, D. L. The genetics of obsessive-compulsive disorder: a review. Dialogues Clin Neurosci 12, 149-63 (2010).

10. Rauch, S. L. et al. Predictors of fluvoxamine response in contamination-related obsessive compulsive disorder: a PET symptom provocation study. Neuropsychopharmacology $\square$ : official publication of the American College of Neuropsychopharmacology 27, 782-91 (2002). 
11. Ahmari, S. E. et al. Repeated cortico-striatal stimulation generates persistent OCD-like behavior. Science 340, 1234-9 (2013).

12. Burguiere, E., Monteiro, P., Feng, G. \& Graybiel, A. M. Optogenetic stimulation of lateral orbitofronto-striatal pathway suppresses compulsive behaviors. Science 340, 1243-6 (2013).

13. Piantadosi, S. C. \& Ahmari, S. E. Using Optogenetics to Dissect the Neural Circuits Underlying OCD and Related Disorders. Curr Treat Options Psychiatry 2, 297-311 (2015).

14. Manning, E. E., Geramita, M. A., Piantadosi, S. C., Pierson, J. L. \& Ahmari, S. E. Distinct patterns of abnormal lateral orbitofrontal cortex activity during compulsive grooming and reversal learning normalize after fluoxetine. Biological Psychiatry 0, (2021).

15. Kravitz, A. V. et al. Regulation of parkinsonian motor behaviours by optogenetic control of basal ganglia circuitry. Nature 466, 622-6 (2010).

16. Albin, R. L., Young, A. B. \& Penney, J. B. The functional anatomy of basal ganglia disorders. Trends Neurosci 12, 366-75 (1989).

17. Alexander, G. E., DeLong, M. R. \& Strick, P. L. Parallel organization of functionally segregated circuits linking basal ganglia and cortex. Annual review of neuroscience 9, 357-81 (1986).

18. Saxena, S., Brody, A. L., Schwartz, J. M. \& Baxter, L. R. Neuroimaging and frontal-subcortical circuitry in obsessive-compulsive disorder. The British journal of psychiatry. Supplement 26-37 (1998).

19. Parker, J. G. et al. Diametric neural ensemble dynamics in parkinsonian and dyskinetic states. Nature 557, 177-182 (2018).

20. Perez, X. A., Zhang, D., Bordia, T. \& Quik, M. Striatal D1 medium spiny neuron activation induces dyskinesias in parkinsonian mice. Movement disorders $\square$ : official journal of the Movement Disorder Society $32,538-548(2017)$.

21. Farre, D. et al. Stronger Dopamine D1 Receptor-Mediated Neurotransmission in Dyskinesia. Molecular neurobiology 52, 1408-1420 (2015). 
22. Thiele, S. L. et al. Selective loss of bi-directional synaptic plasticity in the direct and indirect striatal output pathways accompanies generation of parkinsonism and I-DOPA induced dyskinesia in mouse models. Neurobiology of disease 71, 334-44 (2014).

23. Feyder, M., Bonito-Oliva, A. \& Fisone, G. L-DOPA-Induced Dyskinesia and Abnormal Signaling in Striatal Medium Spiny Neurons: Focus on Dopamine D1 Receptor-Mediated Transmission. Frontiers in behavioral neuroscience 5, 71 (2011).

24. Calabresi, P., Di Filippo, M., Ghiglieri, V. \& Picconi, B. Molecular mechanisms underlying levodopa-induced dyskinesia. Movement disorders $\square$ : official journal of the Movement Disorder Society 23 Suppl 3, S570-9 (2008).

25. Wang, W. et al. Striatopallidal dysfunction underlies repetitive behavior in Shank3-deficient model of autism. The Journal of clinical investigation 127, 1978-1990 (2017).

26. Ade, K. K. et al. Increased Metabotropic Glutamate Receptor 5 Signaling Underlies ObsessiveCompulsive Disorder-like Behavioral and Striatal Circuit Abnormalities in Mice. Biol Psychiatry (2016) doi:10.1016/j.biopsych.2016.04.023.

27. Wan, Y. et al. Circuit-selective striatal synaptic dysfunction in the Sapap3 knockout mouse model of obsessive-compulsive disorder. Biological psychiatry 75, 623-30 (2014).

28. Welch, J. M. et al. Cortico-striatal synaptic defects and OCD-like behaviours in Sapap3-mutant mice. Nature 448, 894-900 (2007).

29. Manning, E. E., Dombrovski, A. Y., Torregrossa, M. M. \& Ahmari, S. E. Impaired instrumental reversal learning is associated with increased medial prefrontal cortex activity in Sapap3 knockout mouse model of compulsive behavior. Neuropsychopharmacology 44, 1494-1504 (2019).

30. Chen, M. et al. Sapap3 deletion anomalously activates short-term endocannabinoid-mediated synaptic plasticity. The Journal of neuroscience $\square$ : the official journal of the Society for Neuroscience 31, 9563-73 (2011). 
31. Wan, Y., Feng, G. \& Calakos, N. Sapap3 deletion causes mGluR5-dependent silencing of AMPAR synapses. The Journal of neuroscience $\square$ : the official journal of the Society for Neuroscience 31, 16685-91 (2011).

32. Corbit, V. L., Manning, E. E., Gittis, A. H. \& Ahmari, S. E. Strengthened inputs from secondary motor cortex to striatum in a mouse model of compulsive behavior. J Neurosci (2019) doi:10.1523/JNEUROSCI.1728-18.2018.

33. Mintzopoulos, D. et al. Striatal magnetic resonance spectroscopy abnormalities in young adult SAPAP3 knockout mice. Biological psychiatry $\square$ : cognitive neuroscience and neuroimaging 1, 3948 (2016).

34. Zhou, P. et al. Efficient and accurate extraction of in vivo calcium signals from microendoscopic video data. elife 7, e28728 (2018).

35. Resendez, S. L. et al. Visualization of cortical, subcortical and deep brain neural circuit dynamics during naturalistic mammalian behavior with head-mounted microscopes and chronically implanted lenses. Nat Protoc 11, 566-597 (2016).

36. Jimenez, J. C. et al. Anxiety Cells in a Hippocampal-Hypothalamic Circuit. Neuron 97, 670-683 e6 (2018).

37. Starr, B. S. \& Starr, M. S. Differential effects of dopamine D1 and D2 agonists and antagonists on velocity of movement, rearing and grooming in the mouse. Implications for the roles of D1 and D2 receptors. Neuropharmacology 25, 455-63 (1986).

38. Molloy, A. G. \& Waddington, J. L. Pharmacological characterization in the rat of grooming and other behavioural responses to the D1 dopamine receptor agonist R-SK\&F 38393. Journal of psychopharmacology (Oxford, England) 1, 177-83 (1987).

39. Page, S. J. \& Terry, P. Conditioned grooming induced by the dopamine D1-like receptor agonist SKF 38393 in rats. Pharmacology, biochemistry, and behavior 57, 829-33 (1997). 
40. Taylor, J. L., Rajbhandari, A. K., Berridge, K. C. \& Aldridge, J. W. Dopamine receptor modulation of repetitive grooming actions in the rat: potential relevance for Tourette syndrome. Brain research 1322, 92-101 (2010).

41. Aldridge, J. W. \& Berridge, K. C. Coding of serial order by neostriatal neurons: a 'natural action' approach to movement sequence. The Journal of neuroscience $\square$ : the official journal of the Society for Neuroscience 18, 2777-87 (1998).

42. Aldridge, J. W., Berridge, K. C. \& Rosen, A. R. Basal ganglia neural mechanisms of natural movement sequences. Canadian journal of physiology and pharmacology 82, 732-9 (2004).

43. Namboodiri, V. M. K. et al. Single-cell activity tracking reveals that orbitofrontal neurons acquire and maintain a long-term memory to guide behavioral adaptation. Nature Neuroscience 22, 11101121 (2019).

44. Markowitz, J. E. et al. The Striatum Organizes 3D Behavior via Moment-to-Moment Action Selection. Cell 174, 44-58.e17 (2018).

45. Barbera, G. et al. Spatially Compact Neural Clusters in the Dorsal Striatum Encode Locomotion Relevant Information. Neuron 92, 202-213 (2016).

46. Soomro, G. M., Altman, D., Rajagopal, S. \& Oakley-Browne, M. Selective serotonin re-uptake inhibitors (SSRIs) versus placebo for obsessive compulsive disorder (OCD). The Cochrane database of systematic reviews CD001765 (2008) doi:10.1002/14651858.CD001765.pub3.

47. Pigott, T. A. \& Seay, S. M. A review of the efficacy of selective serotonin reuptake inhibitors in obsessive-compulsive disorder. J Clin Psychiatry 60, 101-6 (1999).

48. Pittenger, C., Kelmendi, B., Bloch, M., Krystal, J. H. \& Coric, V. Clinical treatment of obsessive compulsive disorder. Psychiatry 2, 34-43 (2005).

49. Bloch, M. H., McGuire, J., Landeros-Weisenberger, A., Leckman, J. F. \& Pittenger, C. Metaanalysis of the dose-response relationship of SSRI in obsessive-compulsive disorder. Mol Psychiatry 15, 850-855 (2010). 
50. Shanahan, N. A. et al. Chronic reductions in serotonin transporter function prevent 5-HT1Binduced behavioral effects in mice. Biol Psychiatry 65, 401-8 (2009).

51. Shmelkov, S. V. et al. Slitrk5 deficiency impairs corticostriatal circuitry and leads to obsessivecompulsive-like behaviors in mice. Nature medicine 16, 598-602, 1p following 602 (2010).

52. Dulawa, S. C., Holick, K. A., Gundersen, B. \& Hen, R. Effects of chronic fluoxetine in animal models of anxiety and depression. Neuropsychopharmacology 29, 1321-30 (2004).

53. Ahmari, S. E. \& Dougherty, D. D. Dissecting Ocd Circuits: From Animal Models to Targeted Treatments. Depress Anxiety (2015) doi:10.1002/da.22367.

54. Nakao, T. et al. Brain activation of patients with obsessive-compulsive disorder during neuropsychological and symptom provocation tasks before and after symptom improvement: a functional magnetic resonance imaging study. Biological psychiatry 57, 901-10 (2005).

55. Ramírez-Armenta, K. I. et al. Optogenetic inhibition of indirect pathway neurons in the dorsomedial striatum reduces excessive grooming in Sapap3-knockout mice. Neuropsychopharmacol. 1-11 (2021) doi:10.1038/s41386-021-01161-9.

56. Bock, R. et al. Strengthening the accumbal indirect pathway promotes resilience to compulsive cocaine use. Nat Neurosci 16, 632-638 (2013).

57. Roltsch Hellard, E. et al. Optogenetic control of alcohol-seeking behavior via the dorsomedial striatal circuit. Neuropharmacology 155, 89-97 (2019).

58. Lobo, M. K. \& Nestler, E. The Striatal Balancing Act in Drug Addiction: Distinct Roles of Direct and Indirect Pathway Medium Spiny Neurons. Frontiers in Neuroanatomy 5, 41 (2011).

59. Kalueff, A. V. et al. Neurobiology of rodent self-grooming and its value for translational neuroscience. Nature reviews. Neuroscience 17, 45-59 (2016).

60. Lamothe, H., Schreiweis, C., Lavielle, O., Mallet, L. \& Burguière, E. Not only compulsivity: The SAPAP3-KO mouse reconsidered as a comorbid model expressing a spectrum of pathological repetitive behaviors. 2020.01.22.915215 
https://www.biorxiv.org/content/10.1101/2020.01.22.915215v2

doi:10.1101/2020.01.22.915215.

61. Rothwell, P. E. et al. Input- and Output-Specific Regulation of Serial Order Performance by Corticostriatal Circuits. Neuron 88, 345-56 (2015).

62. Davis, G. L. et al. Ketamine increases activity of a fronto-striatal projection that regulates compulsive behavior in SAPAP3 knockout mice. Nat Commun 12, 6040 (2021).

63. Stroup, T. S. \& Gray, N. Management of common adverse effects of antipsychotic medications. World Psychiatry 17, 341-356 (2018).

64. Cazorla, M. et al. Dopamine D2 receptors regulate the anatomical and functional balance of basal ganglia circuitry. Neuron 81, 153-64 (2014).

65. Saunders, A. et al. A direct GABAergic output from the basal ganglia to frontal cortex. Nature 521, 85-9 (2015).

66. Kupchik, Y. M. et al. Coding the direct/indirect pathways by D1 and D2 receptors is not valid for accumbens projections. Nature neuroscience 18, 1230-2 (2015).

67. Miyachi, S., Hasegawa, Y. T. \& Gerfen, C. R. Coincident stimulation of convergent cortical inputs enhances immediate early gene induction in the striatum. Neuroscience 134, 1013-1022 (2005).

68. Berretta, S., Parthasarathy, H. B. \& Graybiel, A. M. Local Release of GABAergic Inhibition in the Motor Cortex Induces Immediate-Early Gene Expression in Indirect Pathway Neurons of the Striatum. J Neurosci 17, 4752-4763 (1997).

69. Hadjas, L. C. et al. Projection-specific deficits in synaptic transmission in adult Sapap3-knockout mice. Neuropsychopharmacol. 45, 2020-2029 (2020).

70. Berke, J. D. Functional properties of striatal fast-spiking interneurons. Frontiers in systems neuroscience 5, 45 (2011).

71. Klaus, A. et al. Striatal fast-spiking interneurons: from firing patterns to postsynaptic impact. Frontiers in systems neuroscience 5, 57 (2011). 
72. Tepper, J. M. \& Bolam, J. P. Functional diversity and specificity of neostriatal interneurons. Current opinion in neurobiology 14, 685-92 (2004).

73. Corbit, V. L. et al. Dissociable roles of central striatum and anterior lateral motor area in initiating $\begin{array}{llll}\text { and } & \text { sustaining } & \text { naturalistic } & \text { behavior. }\end{array}$ https://www.biorxiv.org/content/10.1101/2020.01.08.899070v3 doi:10.1101/2020.01.08.899070.

74. Gittis, A. H., Nelson, A. B., Thwin, M. T., Palop, J. J. \& Kreitzer, A. C. Distinct roles of GABAergic interneurons in the regulation of striatal output pathways. The Journal of neuroscience $\square$ : the official journal of the Society for Neuroscience 30, 2223-34 (2010).

75. Zhong, P. \& Yan, Z. Differential regulation of the excitability of prefrontal cortical fast-spiking interneurons and pyramidal neurons by serotonin and fluoxetine. PloS one 6, e16970 (2011).

76. Blomeley, C. P. \& Bracci, E. Serotonin excites fast-spiking interneurons in the striatum. The European journal of neuroscience 29, 1604-14 (2009).

77. Delle Chiaie, R., Scarciglia, P., Pasquini, M., Caredda, M. \& Biondi, M. Aripiprazole Augmentation in Patients with Resistant Obsessive Compulsive Disorder: a Pilot Study. Clin Pract Epidemiol Ment Health 7, 107-111 (2011).

78. Gong, S. et al. Targeting Cre recombinase to specific neuron populations with bacterial artificial chromosome constructs. J Neurosci 27, 9817-9823 (2007).

79. Ghosh, K. K. et al. Miniaturized integration of a fluorescence microscope. Nature methods 8, 8718 (2011). 